\title{
Numerical and experimental analysis of local flow phenomena in laminar Taylor flow in a square mini-channel
}

\author{
C. J. Falconi, ${ }^{1, \text { a) }}$ C. Lehrenfeld, ${ }^{2, b)}$ H. Marschall, ${ }^{3}$ C. Meyer,${ }^{4}$ R. Abiev,${ }^{5}$ \\ D. Bothe ${ }^{3}$ A. Reusken, ${ }^{2}$ M. Schlüter, ${ }^{4}$ and M. Wörner ${ }^{1, c)}$ \\ ${ }^{1}$ Institute of Catalysis Research and Technology, Karlsruhe Institute of Technology, \\ Karlsruhe, Germany \\ ${ }^{2}$ Institut für Geometrie und Praktische Mathematik, RWTH Aachen, Aachen, Germany \\ ${ }^{3}$ Center of Smart Interfaces, Technical University Darmstadt, Darmstadt, Germany \\ ${ }^{4}$ Institute of Multiphase Flows, Technical University Hamburg-Harburg, Hamburg, Germany \\ ${ }^{5}$ St. Petersburg State Institute of Technology, St. Petersburg, Russia
}

(Received 12 March 2015; accepted 21 December 2015; published online 12 January 2016)

\begin{abstract}
The vertically upward Taylor flow in a small square channel (side length $2 \mathrm{~mm}$ ) is one of the guiding measures within the priority program "Transport Processes at Fluidic Interfaces" (SPP 1506) of the German Research Foundation (DFG). This paper presents the results of coordinated experiments and three-dimensional numerical simulations (with three different academic computer codes) for typical local flow parameters (bubble shape, thickness of the liquid film, and velocity profiles) in different cutting planes (lateral and diagonal) for a specific co-current Taylor flow. For most quantities, the differences between the three simulation results and also between the numerical and experimental results are below a few percent. The experimental and computational results consistently show interesting three-dimensional flow effects in the rear part of the liquid film. There, a local back flow of liquid occurs in the fixed frame of reference which leads to a temporary reversal of the direction of the wall shear stress during the passage of a Taylor bubble. Notably, the axial positions of the region with local backflow and those of the minimum vertical velocity differ in the lateral and the diagonal liquid films. By a thorough analysis of the fully resolved simulation results, this previously unknown phenomenon is explained in detail and, moreover, approximate criteria for its occurrence in practical applications are given. It is the different magnitude of the velocity in the lateral film and in the corner region which leads to azimuthal pressure differences in the lateral and diagonal liquid films and causes a slight deviation of the bubble from the rotational symmetry. This deviation is opposite in the front and rear parts of the bubble and has the mentioned significant effects on the local flow field in the rear part of the liquid film. (O) 2016 AIP Publishing LLC. [http://dx.doi.org/10.1063/1.4939498]
\end{abstract}

\section{INTRODUCTION}

For interface resolving simulation of two-phase flows, various numerical methods are available. ${ }^{1-4}$ These encompass nowadays well-established methods such as the volume-of-fluid method, the level-set method, the front-tracking method, as well as emerging methods such as the lattice Boltzmann method. Among other areas of fundamental research, these methods are attractive to study two-phase flows in microfluidics and micro-process engineering numerically. In such applications, the gas/liquid or liquid/liquid flows are predominantly laminar and the number of bubbles or drops is sufficiently small to be computationally manageable, see, e.g., the work of Wörner ${ }^{5}$ for a recent review.

\footnotetext{
a) Present address: Automotive Simulation Center Stuttgart e.V., Stuttgart, Germany.

b) Present address: Institute for Computational and Applied Mathematics, University Münster, Münster, Germany.

c) Author to whom correspondence should be addressed. Electronic mail: martin.woerner@kit.edu
} 
An important point for the assessment of the accuracy of these numerical methods and their further advancement towards valuable tools for engineering applications is the absence of local experimental data for detailed validation on practical flow problems. Additional difficulties in many technical applications are caused by contaminants which may influence or even dominate the behavior of moving interfaces.

A prototypical problem for two-phase flows in narrow channels is the Taylor flow. It is sometimes also referred to as bubble-train flow, segmented flow, or capillary slug flow ${ }^{6}$ and consists of a regular sequence of elongated gas bubbles which almost fill the entire channel cross section (DumitrescuTaylor bubbles ${ }^{7,8}$ ) and are separated by liquid slugs. Gas-liquid Taylor flow in narrow channels is of interest in micro-process engineering,,${ }^{9,10}$ catalytically coated monolith reactors, ${ }^{11}$ material synthesis, ${ }^{12-14}$ and stimulus of biological cells. ${ }^{15}$ While many experimental and numerical studies on Taylor bubbles or Taylor flow consider circular channels, square channels are potentially of larger technological relevance for certain applications, but are less often studied. There is, however, an increasing interest to investigate bubble train flow and gas-liquid Taylor flow in square or rectangular channels experimentally ${ }^{16-27}$ and by three-dimensional numerical simulations. ${ }^{28-37}$ Recent reviews on gas-liquid Taylor flow are provided by Angeli and Gavriilidis, ${ }^{38}$ Gupta et al..${ }^{39}$ Sobieszuk et al., 40 and Talimi et al. ${ }^{41}$ and the reader is referred to these articles and the references therein for further experimental and numerical studies on this subject.

Within the priority programme "Transport Processes at Fluidic Interfaces" (SPP 1506) of the German Research Foundation (DFG), Taylor bubbles and Taylor flow in a circular and square mini-channel are proposed as physical benchmark problems for interface-resolving simulation methods. ${ }^{37,42,43}$ In the work of Marschall et al. ${ }^{37}$ a combined experimental and numerical study on a single Taylor bubble in a square vertical mini-channel is presented. The comparison focused on the non-axisymmetric bubble shape, where precise profiles were measured in longitudinal cuts in lateral and diagonal directions by synchrotron radiation in conjunction with ultrafast radiographic imaging. ${ }^{27}$ The influence of different numerical methods for interface resolving simulations and different surface tension force approximations is investigated and, in general, a very good agreement between the four considered codes and the experimental interface profile is found (especially as concerns the curvature of the front and rear menisci). ${ }^{37}$ However, some differences occurred with respect to the minimum thickness of the liquid film which separates the bubble from the channel walls. A drawback of this study is that no experimental data on the local velocity field are available for a comparison.

In the present paper, a similar combined experimental and numerical study as in the work of Marschall et al. ${ }^{37}$ is performed but with focus on local features of the velocity field, which is measured within the liquid phase by micro-Particle-Image-Velocimetry ( $\mu$ PIV). Such measurements are still rare, especially within the liquid film. Meyer et $a l .{ }^{44}$ provide a detailed overview on related experimental studies in the literature. In contrast to our previous study, ${ }^{37}$ which dealt with a single Taylor bubble, Taylor flow is considered in the present paper. The liquid slug length is rather short so that the Poiseuille velocity profile is not fully developed and there is a notable interaction between neighboring Taylor bubbles.

As a first goal of our combined experimental and numerical study, we provide quantitative local velocity field information, e.g., velocity profiles for one particular Taylor flow. Obtaining quantitative benchmark results with a single computer code is difficult, since the accuracy of the code must be guaranteed which is hard to achieve for the rather complex Taylor flow in a square channel. Here, we decided to use three academic in-house codes which differ with respect to the numerical method and certain aspects concerning the numerical setup (due to code-internal restrictions). For many quantities like bubble velocity, bubble length, liquid slug length, local bubble radius, and local axial centerline velocity in the liquid slug, the differences between the three simulation results and also between the numerical and experimental results are below a few percent. We consider these simulations' results to be useful quantities for comparison in future fluid dynamic simulations of Taylor flow. Furthermore, the numerical and experimental results reveal some interesting phenomena, namely, a local backflow in the rear part of the liquid film. Such a local backflow in a fixed frame of reference was already found in the numerical simulations of Quan ${ }^{45}$ who studied the effects of a co-current upward and downward flow on a single Taylor bubble in a round capillary with a diameter of $3.2 \mathrm{~cm}$. His results show that the velocity profile in the liquid film is almost independent of the mean velocity of the co-current 
flow. Interestingly, the present results for a square channel show that the axial locations of this local backflow region differ in the lateral film and in the corner film. To the best of our knowledge, this behavior has not been reported in the literature so far.

As a second goal, it is demonstrated how the present integrated experimental and computational study can provide a detailed insight and understanding of this local flow phenomenon. The role of the experiment was to demonstrate that this feature, which is observed in computational fluid dynamics (CFD) simulations under idealized conditions (clean interfaces), is not a numerical artifact but is observed in real Taylor flow. The role of the computations was to provide fully resolved information on the bubble shape, the velocity field, and the pressure field in the entire Taylor flow unit cell (which consists of one Taylor bubble and one liquid slug). Only these data - which cannot be obtained in their completeness nowadays by the even most advanced experimental techniques — provide the information to understand in detail the underlying hydrodynamics and to derive criteria for the occurrence of this phenomenon in practical flow systems.

The paper is organized as follows. Section II gives an overview on the experimental setup and measurement techniques. Section III presents the numerical counterpart. In Section IV, the experimental and numerical results are compared. In Section V, a physical explanation for the local backflow in the liquid film is given and the conditions under which it may occur are discussed. The paper closes with conclusions in Section VI.

\section{EXPERIMENTS}

The present experiment considers a co-current upward Taylor flow in a square vertical minichannel with cross section $2.076 \mathrm{~mm} \times 2.076 \mathrm{~mm}$ and corresponds to scenario "TFSC Small Slug" in the work of Meyer et al. ${ }^{44}$ The liquid phase is a water/glycerol mixture and the gas phase is air. Special care has been given to the generation of an almost ideal Taylor flow, where bubbles of identical volume and shape move with identical velocity and are separated from each other by liquid slugs of identical length. By a special injection valve combined with an extraordinary compensation pipe, it was possible to produce a Taylor flow where the standard deviations in bubble length and slug length are below $3 \%$ and that of the bubble velocity is below $2 \% .{ }^{44}$

For accurate $\mu$ PIV measurements of the velocity in the liquid film, the following procedure is adopted. To achieve precise optical access without refraction caused by curved interfaces, refractive index matching (RIM) is used. The glass capillary is surrounded by another (larger) capillary filled with a mixture of Dimethylsulfoxide (DMSO) and distilled water (volume concentration of DMSO in the DMSO/water mixture is 95\%), see Fig. 1(a). The setup is placed on a compact precision xy-stage (OWIS, PKTM 100) which is traversed in several steps (e.g., $20 \mu \mathrm{m}$ ) to measure the liquid velocity in the lateral film at different positions. To obtain the velocity profile in diagonal direction, several points along the diagonal plane are captured (white dots in Fig. 1(b)). The RIM requires a precise control of the temperature which was set to $20 \pm 0.05^{\circ} \mathrm{C}$. The liquid density, liquid viscosity, and

(a)

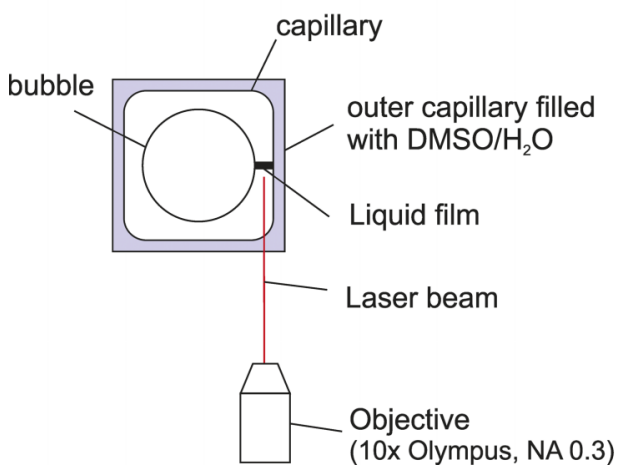

(b)

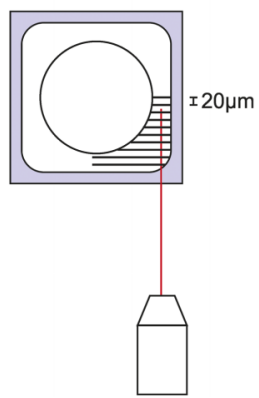

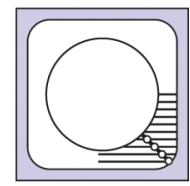

FIG. 1. Cross-sectional view of the experimental test section and setup for the $\mu$ PIV measurements of the velocity inside the liquid film: (a) lateral and (b) diagonal. 
coefficient of surface tension for this temperature are $\rho_{\mathrm{L}}=1197.2 \mathrm{~kg} / \mathrm{m}^{3}, \eta_{\mathrm{L}}=0.0481 \mathrm{~kg} / \mathrm{ms}$, and $\sigma=0.0624 \mathrm{~N} / \mathrm{m}$, respectively. For further details on the experimental setup as well as the instrumentation and measurement technique (including the RIM), see the work of Meyer et al. ${ }^{44}$

The measured bubble velocity is $U_{\mathrm{B}}=135.9 \pm 1.9 \mathrm{~mm} / \mathrm{s}$. The corresponding values of the capillary number and Reynolds number are $C a=\eta_{\mathrm{L}} U_{\mathrm{B}} / \sigma=0.1$ and $R e=\rho_{\mathrm{L}} D_{\mathrm{h}} U_{\mathrm{B}} / \eta_{\mathrm{L}}=7.0$, respectively, where $D_{\mathrm{h}}=2.076 \mathrm{~mm}$ is the hydraulic diameter. The measured values of the total bubble length and liquid slug length are $L_{\mathrm{B}}=3.26 \pm 0.18 \mathrm{~mm}$ and $L_{\mathrm{S}}=1.33 \pm 0.09 \mathrm{~mm}$, respectively, so that the length of the unit cell is $L_{\mathrm{UC}}=L_{\mathrm{B}}+L_{\mathrm{S}}=4.59 \pm 0.27 \mathrm{~mm}$. The gas volume fraction in the unit cell was measured as $\varepsilon=0.37 \pm 0.02$. This value was determined by evaluation of 20 images captured by the high speed camera. The gas volume was evaluated for each image with image processing software for contour detection assuming rotational symmetry with respect to the channel axis. The numerical simulations to be discussed below show a slight deviation of the Taylor bubble from rotational symmetry. However, this deviation is rather small (cf. Section IV A) so that the error for the estimated experimental volume fraction is quite low. From the $\mu$ PIV observations, the liquid film thickness was estimated at different axial positions. These results and the measured velocity profiles in the liquid slug and liquid film will be presented and discussed below in Sections IV C and IV D, respectively.

\section{NUMERICAL SIMULATIONS}

In this section, the governing equations and the assumptions underlying the numerical simulations are provided. The three computer codes are described in detail in the work of Marschall et al. ${ }^{37}$ For this reason, only a brief overview is given in Section III B on the numerical method underlying each code. In Section III C, the computational setup is described and some global simulation results are given.

\section{A. General assumptions and governing equations}

In the numerical simulations, the gas and liquid phases are considered as two incompressible immiscible Newtonian fluids with constant density and viscosity. Both phases are separated by an interface of zero thickness (sharp-interface model). The flow is isothermal and the coefficient of surface tension is constant. The physical properties of the liquid phase and the coefficient of surface tension are the same as in the experiment (cf. Section II). The values of the gas density and viscosity in the simulations are $\rho_{\mathrm{G}}=1.3 \mathrm{~kg} / \mathrm{m}^{3}$ and $\eta_{\mathrm{G}}=2 \times 10^{-5} \mathrm{~kg} / \mathrm{ms}$, respectively.

To compute the incompressible two-phase flow, the continuity equation and linear momentum equation in one-field formulation are solved according to

$$
\nabla \cdot \mathbf{u}=0, \quad \partial_{t}(\rho \mathbf{u})+\nabla \cdot(\rho \mathbf{u u})=-\nabla p+\nabla \cdot \boldsymbol{\tau}+\rho \mathbf{g}+\mathbf{f}_{\Sigma} .
$$

Herein, $\mathbf{u}$ is the continuous velocity field, $\boldsymbol{\tau}=\eta\left(\nabla \mathbf{u}+(\nabla \mathbf{u})^{\mathrm{T}}\right)$ is the viscous stress tensor, $\mathbf{f}_{\Sigma}=\sigma \kappa \mathbf{n}_{\Sigma} \delta_{\Sigma}$ is the volumetric interfacial force density due to surface tension, $\mathbf{n}_{\Sigma}$ is the interface unit normal vector pointing into the gas phase, $\kappa=-\nabla_{\Sigma} \cdot \mathbf{n}_{\Sigma}$ is the (total) interface curvature, and $\delta_{\Sigma}$ is a Dirac distribution associated with the interface. The set of Equations (1) is complemented by an advection equation for interface capturing which is method-dependent and, hence, mentioned below.

\section{B. Numerical methods and computer codes}

\section{FS3D}

The volume-of-fluid in-house code $\mathrm{FS}^{4} \mathrm{D}^{46}$ is based on a finite volume discretization and solves for the two-phase Navier-Stokes equations along with the phase fraction advection equation adopting the CGS system of units. The interface is kept sharp during simulations by geometrically reconstructing and advecting the interface, adopting the Piecewise Linear Interface Calculation (PLIC) method ${ }^{47-49}$ and a split advection algorithm. ${ }^{46}$ The underlying structured Cartesian grid supports staggered variable arrangement with the velocity field being stored on cell faces and the pressure field on cell centers. The code is massively parallelized using MPI and OpenMP.

The name FS3D means Free Surface 3D. The code is written in Fortran being actively developed at ITLR (Univ. Stuttgart) and MMA (Center of Smart Interfaces, Technische Univ. Darmstadt). The 
code has been extensively validated, in particular, for hydrodynamics and mass transfer of single rising bubbles with and without reaction, ${ }^{50,51}$ Newtonian and non-Newtonian droplet collision, ${ }^{52,53}$ and for hydrodynamics of falling films,${ }^{54}$ in the course of which its discretization practice and numerical method have proven accurate and reliable.

\section{TURBIT-VOF}

The TURBIT-VOF code solves the locally volume-averaged two-phase Navier-Stokes equation in non-dimensional single field formulation for two incompressible Newtonian fluids on a regular staggered Cartesian mesh by a finite volume method. In each mesh cell containing both phases, the interface is represented by a PLIC plane of zero thickness. The interface evolution is described by the advection equation for the liquid volume fraction. This equation is solved by a volume-of-fluid method which consists of two steps. In the first step, the interface location is geometrically reconstructed by an in-house PLIC algorithm called EPIRA. ${ }^{55}$ On a 3D structured orthogonal non-equidistant fixed grid, it reconstructs a planar interface of any orientation exactly. In the second step, the fluxes of the liquid over the cell faces are computed by an unsplit advection scheme. Time integration of the momentum equation is performed by an explicit third-order Runge-Kutta method. For approximation of spatial derivatives, second-order central difference schemes are used. A divergence free velocity field is ensured at the end of each time step by a projection method. Further details can be found in the work of Öztaskin $e t a l .{ }^{32}$

\section{DROPS}

The three-dimensional finite element software package DROPS uses an interface capturing approach based on a level-set formulation. An adaptive multilevel mesh hierarchy allows for an efficient resolution of the important flow features on unstructured tetrahedral meshes.

In the level-set method, a scalar function $\varphi=\varphi(\mathbf{x}, t)$, denoted as the level-set function, with the property $\varphi(\mathbf{x}, t)>0$ if $\mathbf{x} \in \Omega_{2}(t), \varphi(\mathbf{x}, t)<0$ if $\mathbf{x} \in \Omega_{1}(t)$, and $\varphi(\mathbf{x}, t)=0$ if $\mathbf{x} \in \Sigma(t)$, is used to describe the position of the interface. As in the volume-of-fluid method, this description of the interface by Eq. (2) is only implicit,

$$
\partial_{t} \varphi+\mathbf{u} \cdot \nabla \varphi=0, \quad t \geq 0, \quad \mathbf{x} \in \Omega .
$$

For the spatial discretization of velocity and pressure, the LBB-stable Hood-Taylor finite element pair is used. To account for discontinuities in the pressure field, the pressure space is enriched using an extended finite element (XFEM) space and a modified Laplace-Beltrami technique is used to describe surface tension accurately. ${ }^{56}$ For the discretization of the level-set function, continuous piecewise quadratics combined with streamline diffusion stabilization are used. For the time discretization, a backward Euler scheme is used where the nonlinearities are resolved using (modified) fixed point iteration schemes. For a detailed description, the reader is referred to Groß et al. ${ }^{57}$ Bertakis et al.,${ }^{58}$ and Groß and Reusken, ${ }^{4}$ and to the DROPS internet page (http://www.igpm.rwth-aachen.de/DROPS/). Note that some preliminary results of the DROPS code for this test case are already published in the work of Meyer et al. ${ }^{44}$

\section{Numerical setup and global results}

The setup for the numerical simulations is chosen in accordance with the experimental conditions given in Section II. The Taylor flow is represented by a single unit cell in combination with periodic boundary conditions in axial direction and no-slip boundary conditions at the four lateral channel walls, see Fig. 2 for a sketch and the coordinate system. The size of the Taylor flow unit cell is $D_{\mathrm{h}} \times D_{\mathrm{h}} \times L_{\mathrm{UC}}$ with $D_{\mathrm{h}}=2.076 \mathrm{~mm}$ and $L_{\mathrm{UC}}=4.59 \mathrm{~mm}$. The volume of the Taylor bubble is $V_{\mathrm{B}}=7.424 \mathrm{~mm}^{3}$ which corresponds to a gas volume fraction in the unit cell of $\varepsilon=V_{\mathrm{B}} / V_{\mathrm{uc}}=0.375$.

The initial bubble shape differs from code to code. In FS3D, it is a rectangular cuboid. In DROPS, it is a cylindrical interface with two half-spheres at the top and bottom with the correct initial volume. In TURBIT-VOF, a semi-analytical axisymmetric profile is employed ${ }^{59}$ which is based on the potential flow solution of Dumitrescu. ${ }^{7}$ The initial conditions for the velocity field are as follows. 


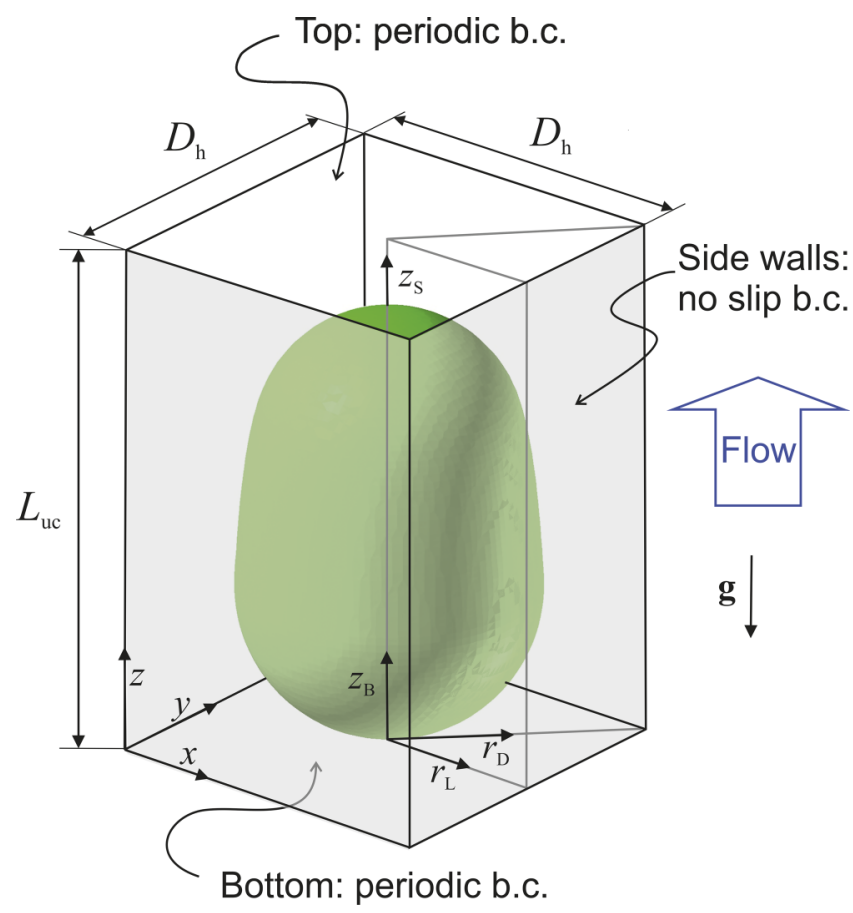

FIG. 2. Sketch of computational setup and coordinate system for Taylor flow in a square channel. The longitudinal cuts where data are evaluated in lateral and diagonal directions are also presented.

In FS3D, the initial axial velocity is set to the experimental bubble velocity and is uniform in the entire computational domain. In DROPS, both phases are initially at rest. In TURBIT-VOF, the initial velocity profile is parabolic within the channel cross section and is axially uniform. The interest in this paper is on the quasi-steady solution and it is assumed that the initial values have only influence on the computational robustness and time but not on the solution itself.

To make the pressure field compatible with the periodic boundary conditions, from the physical pressure $p$, a part is subtracted which decreases linearly with increasing vertical coordinate and corresponds to the pressure drop per unit cell length. ${ }^{28,32}$ The periodic part of the pressure $\hat{p}$ serves as an unknown and is determined by solving a pressure Poisson equation in order to obtain a divergence-free velocity field. The linear decreasing pressure part transforms to a constant uniform source term in the axial component of the Navier-Stokes equation. In DROPS and TURBIT-VOF, this term drives the flow and its value is adjusted such that, finally, the target value for the experimental bubble velocity $U_{\mathrm{B}}=135.9 \mathrm{~mm} / \mathrm{s}$ is achieved. In FS3D, the volumetric flow rate is prescribed and the pressure drop adjusts accordingly. While all computations are time-dependent, here only results of the quasi-steady state are discussed where the bubble velocity, bubble shape, and the liquid superficial velocity are constant in time. The respective stop criteria for the three codes are as follows. For TURBIT-VOF, the simulation is stopped when in a sufficiently long time interval the temporal change of the bubble velocity and the maximum bubble diameter were both below certain limits, namely, $0.1 \mathrm{~mm} / \mathrm{s}$ and $0.5 \mu \mathrm{m}$. The DROPS simulations have been stopped when the change of the bubble velocity was below $1 \%$ and the changes of the bubble length and maximal diameter were below $0.5 \%$. The FS3D simulations have been stopped when the change in bubble rising velocity was below $5 \mu \mathrm{m} / \mathrm{s}$.

The computational domain differs in the three codes. In TURBIT-VOF, it is equivalent to the unit cell. In FS3D and DROPS, one quarter and one eighth of the channel cross section is considered, respectively, in combination with appropriate symmetry boundary conditions. For specifying a suitable grid resolution, we took advantage from the experience gained by our previous study on the flow of a Taylor bubble. ${ }^{37}$ FS3D and TURBIT-VOF use a Cartesian grid and Table I lists the equivalent number of mesh cells for the entire unit cell. DROPS uses an unstructured locally refined grid and the resolution given in Table I corresponds to that of a Cartesian grid for the entire unit cell. These 
TABLE I. Computational domain, equivalent grid resolution for the entire unit cell, and integral simulation results for the three computer codes.

\begin{tabular}{lcccr}
\hline \hline & FS3D & TURBIT-VOF & DROPS & Experiment \\
\hline Domain size & $V_{\mathrm{uc}} / 4$ & $V_{\mathrm{uc}}$ & $V_{\mathrm{uc}} / 8$ & \\
Grid & $64 \times 64 \times 128$ & $100 \times 100 \times 220$ & $64 \times 64 \times 128$ & $135.9 \pm 1.9$ \\
Bubble velocity $(\mathrm{mm} / \mathrm{s})$ & 135.9 & 135.9 & 135.0 & $3.26 \pm 0.18$ \\
Bubble length $(\mathrm{mm})$ & 3.355 & 3.328 & 3.329 & $1.33 \pm 0.09$ \\
Liquid slug length $(\mathrm{mm})$ & 1.235 & 1.262 & 1.261 & 121.2 \\
Pressure drop $(\mathrm{Pa})$ & 117.8 & 119.2 & 382.6 & \\
Volumetric flow rate $\left(\mathrm{mm}^{3} / \mathrm{s}\right)$ & 377.2 & 391.6 & \\
\hline \hline
\end{tabular}

grids correspond to a resolution of the minimum lateral film thickness (which is considered to be the smallest length scale of the flow) by about 3 mesh cells. In addition, simulations on a coarser grid were performed with TURBIT-VOF and DROPS. Based on these preliminary simulations, the results presented below for both codes are considered to have a numerical error (in velocity, pressure, and interface location) of at most a few percent. Due to tremendous computational costs for further (space and time) resolution increase, we are neither able to accomplish comprehensive mesh-independence studies nor can we provide sound estimates of convergence rates on the basis of the presented Taylor flow simulations. In fact, this is the main reason for using three different codes, in order to assess the accuracy of the simulation results.

Table I lists the values of the bubble velocity $U_{\mathrm{B}}$, the bubble length $L_{\mathrm{B}}$, the liquid slug length $L_{\mathrm{S}}$, the axial pressure drop along the unit cell, and the volumetric flow rate of the gas-liquid flow. To quantify deviations of the results, the following procedure is adopted. The relative deviation between computational results is computed as the maximum absolute deviation from the mean value of the three codes normalized by the mean value. The relative deviation between experimental and numerical data is computed as the absolute value of the difference between the mean computational and experimental value, normalized by the experimental value. Table I shows that for all codes, the numerical values for $U_{\mathrm{B}}$ and $L_{\mathrm{B}}$ are within the experimental uncertainty. The deviation between the three codes for both quantities is less than $0.5 \%$. Notably, the computed value of $L_{\mathrm{B}}$ is for all codes higher than the nominal experimental value. The axial pressure drop and the volumetric flow rate have not been measured. For both quantities, the deviation between the codes is less than $2 \%$. The total superficial velocity is about $J=88-91 \mathrm{~mm} / \mathrm{s}$ and the volumetric flow rate ratio is about $\dot{V}_{\mathrm{G}} /\left(\dot{V}_{\mathrm{G}}+\dot{V}_{\mathrm{L}}\right)=0.57$.

Fig. 2 shows the locations where quantities are evaluated and compared with experimental data and the associated local coordinate system. For presenting the results in terms of local profiles, appropriate non-dimensional coordinates are introduced. In radial direction, the distance from the channel axis in lateral and diagonal directions is defined as $\tilde{r}_{\mathrm{L}}=r_{\mathrm{L}} / D_{\mathrm{h}}$ and $\tilde{r}_{\mathrm{D}}=r_{\mathrm{D}} / D_{\mathrm{h}}$, respectively. In axial direction, the vertical distance from the rear meniscus of the bubble is defined as $\tilde{z}_{\mathrm{B}}=z_{\mathrm{B}} / L_{\mathrm{B}}$, and for the liquid slug the vertical distance from the bubble front meniscus as $\tilde{z}_{\mathrm{S}}=z_{\mathrm{S}} / L_{\mathrm{S}}$.

For comparison of the location of the backflow regions, in addition to the above base case, one further simulation is carried out for a higher capillary number where the Taylor bubble is axisymmetric. This simulation is performed with TURBIT-VOF for the same setup and fluid properties as given above. Only the pressure drop along the unit cell is increased to $168.2 \mathrm{~Pa}$ which results in a larger thickness of the liquid film. This simulation is, therefore, performed on a coarser grid consisting of $50 \times 50 \times 110$ mesh cells. The respective quasi-steady simulation results of this case (denoted in the sequel as comparative case) are as follows: $U_{\mathrm{B}}=293.1 \mathrm{~mm} / \mathrm{s}, L_{\mathrm{B}}=3.699 \mathrm{~mm}, L_{\mathrm{S}}=0.891 \mathrm{~mm}$, $C a=0.23$, and $R e=15.1$.

\section{RESULTS}

This section starts with results for the shape of the Taylor bubble. This is followed by a discussion of the streamlines and the flow within the gas bubble. Finally, a comparison of the measured and computed velocity profiles in the liquid slug and liquid film is presented. In this section, only the base case is considered; results of the comparative case will be discussed in Section V. 


\section{A. Bubble shape and liquid film thickness}

In the experiment, the bubble radius and liquid film thickness in a horizontal cross section were not measured directly. Instead, their values were estimated from $\mu$ PIV observations, however, only for the lateral cut. The respective values are listed in Table II for the axial positions $\tilde{z}_{\mathrm{B}}=0.25,0.5$, and 0.75 . Also given is the computed bubble radius and liquid film thickness in lateral and diagonal directions for the three codes. The latter data are obtained by different post-processing procedures. In FS3D, the profile is extracted from the $f=0.5$ isosurface of the liquid volume fraction; in DROPS, it is obtained from the location where the level-set function is zero; and in TURBIT-VOF, it is extracted from the geometrically reconstructed interface (set of geometrical centers of the PLIC faces). The relative difference of the bubble radius in lateral and diagonal directions between the three codes is below $0.6 \%$ for all three axial positions. The relative difference between the estimated experimental lateral bubble radius and the respective mean value of the three computer codes for the three axial positions is below $2.7 \%$.

Also given in Table II is the maximum bubble radius and the minimum thickness of the liquid film. The deviation in the maximum bubble radius between the three codes is less than $0.8 \%$. Experiments in circular capillary tubes show that the maximum bubble diameter and the minimum liquid film thickness increase/decrease with increasing bubble length, but become independent from the bubble length for sufficiently long bubbles. ${ }^{60,61}$ Recently, Klaseboer et al ${ }^{62}$ proposed a criterion for the minimum bubble length in circular channels such that "entry and exit effects" are no longer important for the developed film thickness. Evaluation of this criterion for $C a=0.1$ yields $L_{\mathrm{B} \text {,crit }} \approx 4.8 D$. While there is no such criterion for square channels, it is obvious that the present bubble has to be considered as "short." Clearly, an expression is missing in the literature which correlates the maximal bubble diameter not only with $\mathrm{Ca}$ but also with $L_{\mathrm{B}}$, both for circular and square channels. In the absence of such a correlation, the computed maximum bubble diameter in diagonal direction is compared with the following experimental correlation of Kreutzer et al. ${ }^{11}$ for the developed region of long Taylor bubbles in a square channel,

$$
\frac{D_{\mathrm{B}, \mathrm{D}}}{D_{\mathrm{h}}}=0.7+0.5 \exp \left(-2.25 C a^{0.445}\right) .
$$

TABLE II. Bubble radius and liquid film thickness (in $\mu \mathrm{m}$ ) in lateral (L) and diagonal (D) directions at three different axial positions. The positions refer to the distance from the bubble rear normalized by the computed bubble length of each code. Also listed are the maximum bubble radius and the minimum film thickness and the respective axial positions.

\begin{tabular}{|c|c|c|c|c|c|c|}
\hline & Cut & $\tilde{z}_{\mathrm{B}}$ & FS3D & TURBIT-VOF & DROPS & Experiment \\
\hline \multirow[t]{6}{*}{ Bubble radius } & \multirow[t]{3}{*}{$\mathrm{L}$} & 0.25 & 952 & 961 & 960 & $946 \pm 8$ \\
\hline & & 0.50 & 918 & 925 & 922 & $940 \pm 8$ \\
\hline & & 0.75 & 824 & 825 & 824 & $847 \pm 8$ \\
\hline & \multirow[t]{3}{*}{$\mathrm{D}$} & 0.25 & 983 & 991 & 993 & \\
\hline & & 0.50 & 949 & 953 & 954 & \\
\hline & & 0.75 & 839 & 845 & 839 & \\
\hline \multirow[t]{6}{*}{ Film thickness } & \multirow[t]{3}{*}{$\mathrm{L}$} & 0.25 & 86 & 77 & 78 & $92 \pm 8$ \\
\hline & & 0.50 & 120 & 113 & 116 & $98 \pm 8$ \\
\hline & & 0.75 & 214 & 213 & 214 & $191 \pm 9$ \\
\hline & \multirow[t]{3}{*}{$\mathrm{D}$} & 0.25 & 485 & 477 & 475 & \\
\hline & & 0.50 & 519 & 515 & 514 & \\
\hline & & 0.75 & 629 & 623 & 629 & \\
\hline \multirow[t]{6}{*}{ Extreme value } & \multirow[t]{3}{*}{$\mathrm{L}$} & $\tilde{z}_{\mathrm{B}}$ & 0.198 & 0.173 & 0.194 & \\
\hline & & Max. bubble radius & 959 & 973 & 967 & \\
\hline & & Min. film thickness & 79 & 65 & 71 & \\
\hline & \multirow[t]{3}{*}{$\mathrm{D}$} & $\tilde{z}_{\mathrm{B}}$ & 0.273 & 0.270 & 0.275 & \\
\hline & & Max. bubble radius & 985 & 991 & 994 & \\
\hline & & Min. film thickness & 483 & 477 & 474 & \\
\hline
\end{tabular}


Evaluation of Eq. (3) gives a bubble radius of $958 \mu \mathrm{m}$. The present numerical results for the maximum bubble diameter are about $3.3 \%$ larger than this value.

The minimal liquid film thickness and the maximum bubble diameter are directly related. Due to the much smaller dimension of the film thickness, the relative deviation between the codes for this quantity is much larger than for the bubble diameter. While in diagonal direction the difference in the minimum numerical film thickness is only about $1 \%$, it is about $10 \%$ in lateral direction. This relatively large error is probably due to poor spatial resolution of this region (about three mesh cells) which is insufficient to provide high precision results. The deviations for the axial position where the liquid film is thinnest are about $0.3 \%$ and $1.5 \%$ of the bubble length for the lateral and diagonal films, respectively. The smallest minimum film thickness in lateral direction is computed by TURBIT-VOF. Furthermore, the axial position of the minimum film thickness in TURBIT-VOF is somewhat closer to the rear meniscus of the bubble than in FS3D and DROPS. This behavior was already observed in our previous paper on the flow of a single Taylor bubble in a square channel. ${ }^{37}$

To compare the computed shapes of the Taylor bubble in more detail, in Fig. 3, axial profiles of the interface in longitudinal cuts in lateral and diagonal directions are shown. In FS3D, the bubble is slightly longer than in TURBIT-VOF and DROPS. Nevertheless, the computed bubble shapes agree very well. This is especially true for the curvature of the front and rear menisci and for the bubble body. The largest differences occur at the rear part of the bubble at $z / D_{\mathrm{h}} \approx 0.1-0.3$. In this range, TURBIT-VOF predicts a slightly larger bubble diameter as compared to the two other codes (see the insets in Fig. 3). The interfacial profiles in Fig. 3 show that there is no "developed" film region, where

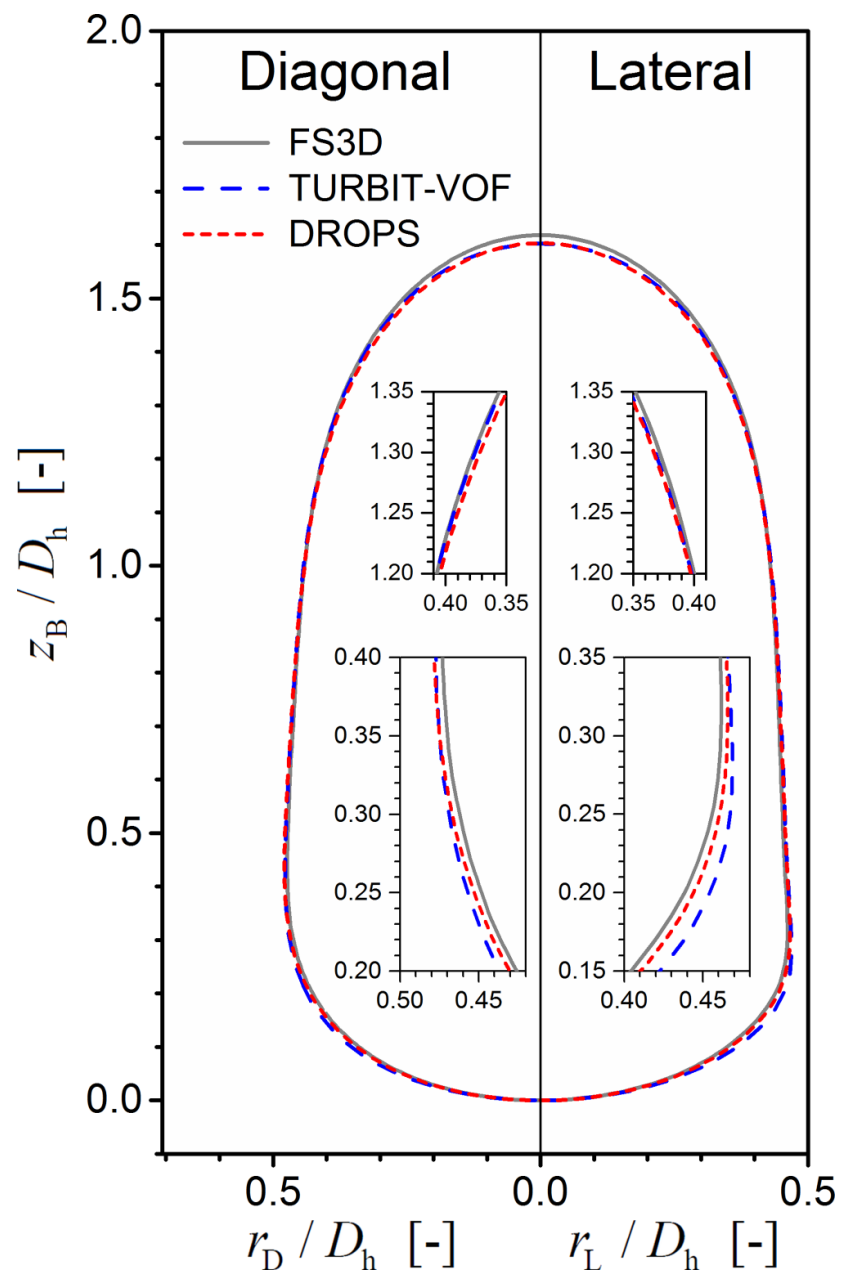

FIG. 3. Profiles of the computed Taylor bubble shape in a longitudinal cut in lateral and diagonal directions. 
the bubble diameter and the liquid film thickness are axially uniform. The latter is a common feature of long Taylor bubbles. In the present case, the Taylor bubble is rather short as the ratio between bubble length and hydraulic diameter is only $L_{\mathrm{B}} / D_{\mathrm{h}} \approx 1.6$, while the ratio between the volume equivalent bubble diameter and $D_{\mathrm{h}}$ is only about 1.17 .

From the interface profiles displayed in Fig. 3, the local ratio of the bubble radius in diagonal and lateral directions is calculated. The axial profiles of this aspect ratio are very similar for all codes and reveal an interesting result. In the front part of the bubble, namely, in the range $0.19<\tilde{z}_{\mathrm{B}}<1$, the bubble radius is larger in the diagonal direction than in the lateral direction so that the crosssectional aspect ratio is larger than unity (cf. Table II). The maximum value is about 1.04 and occurs at $\tilde{z}_{\mathrm{B}} \approx 0.32-0.35$. In the rear part of the bubble, i.e., in the range $0<\tilde{z}_{\mathrm{B}}<0.19$, the value of the cross-sectional aspect ratio is in contrast less than unity. Thus, at the bubble rear, the bubble radius is larger in the lateral direction than in the diagonal one. The smallest value of the aspect ratio is about $0.96-0.97$ and occurs at $\tilde{z}_{\mathrm{B}} \approx 0.09-0.1$. This change of the aspect ratio from values larger than 1 at the bubble front and body to values smaller than 1 at the bubble rear was also observed in the experiments ${ }^{27}$ and computations ${ }^{37}$ of our previous study on a single Taylor bubble in a square channel, where the capillary number was similar $(\mathrm{Ca}=0.088)$ but the Reynolds number was higher $(R e=17.0)$ and the Taylor bubble was much longer.

Of interest is a discussion of the observed deviation of the bubble shape from rotational symmetry in context with the literature results for a semi-infinite bubble that displaces liquid from a square capillary. Kolb and Cerro ${ }^{63}$ performed experiments on the coating of the inside of a square capillary and found that the bubble becomes non-axisymmetric for $C a \approx 0.1$ so that the ratio between the maximum bubble radius in lateral and diagonal directions becomes less than unity for lower values of $\mathrm{Ca}$. This result is consistent with the tip asymmetry found by Hazel and Heil ${ }^{64}$ for the steady propagation of a semi-infinite bubble into a square channel by a finite-element based direct solution of the free-surface Stokes equations. The authors argue that this tip asymmetry is caused by the non-axisymmetric passage of fluid around the front meniscus. In square channels, the fluid tends to

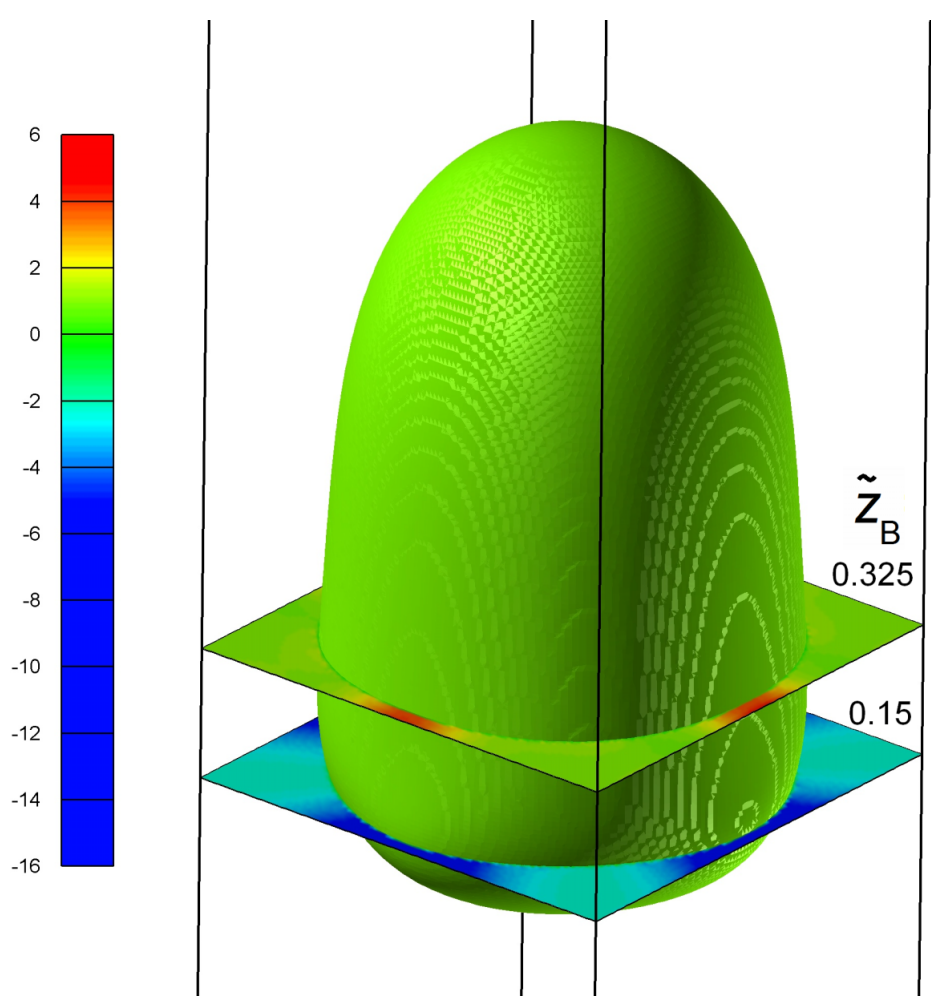

FIG. 4. Computed Taylor bubble shape and contour plot of the (non-dimensional) periodic pressure field $\hat{p}$ in two different horizontal cross sections at $\tilde{z}_{\mathrm{B}}=0.15$ and 0.325 (results of TURBIT-VOF). 
move towards the corners, which offer less resistance to the flow than the thinner regions along the channel sides. The resulting transverse flows induce a transverse pressure gradient that lowers the fluid pressure in the corners, where the air-liquid interface moves radially outwards. ${ }^{64}$

In view of this, contour-plots of the computed (non-dimensional) periodic pressure field $\hat{p}$ in a horizontal cross section are shown in Fig. 4 for two different axial locations (results of TURBIT-VOF). In the cross section at $\tilde{z}_{\mathrm{B}}=0.325$, the pressure at the channel sides is larger than in the corners, in agreement with Hazel and Heil. ${ }^{64}$ In contrast, in the cross section at $\tilde{z}_{\mathrm{B}}=0.15$ (i.e., close to the rear meniscus), the pressure at the channel sides is smaller than in the corners. At each point of the interface, there is in normal direction a balance between capillary, viscous, and pressure forces. Here, normal viscous stresses are expected to be negligible. The non-uniform azimuthal pressure distribution in a horizontal cross section is then locally balanced by azimuthally non-uniform capillary forces. As the coefficient of surface tension is constant in our simulations, this can only be achieved by a change of the local interface curvature which results in the observed deviation of the Taylor bubble shape from rotational symmetry. In Section V, it will be shown that the difference between the maximum bubble radius in lateral and diagonal directions and the axial variation of the bubble aspect ratio has strong implications on the local flow field in the rear part of the liquid film.

\section{B. Streamlines}

Fig. 5 shows streamlines of the flow in a frame of reference moving with the bubble in a lateral and diagonal cut in combination with a contour-plot of the vertical velocity component in a fixed frame of

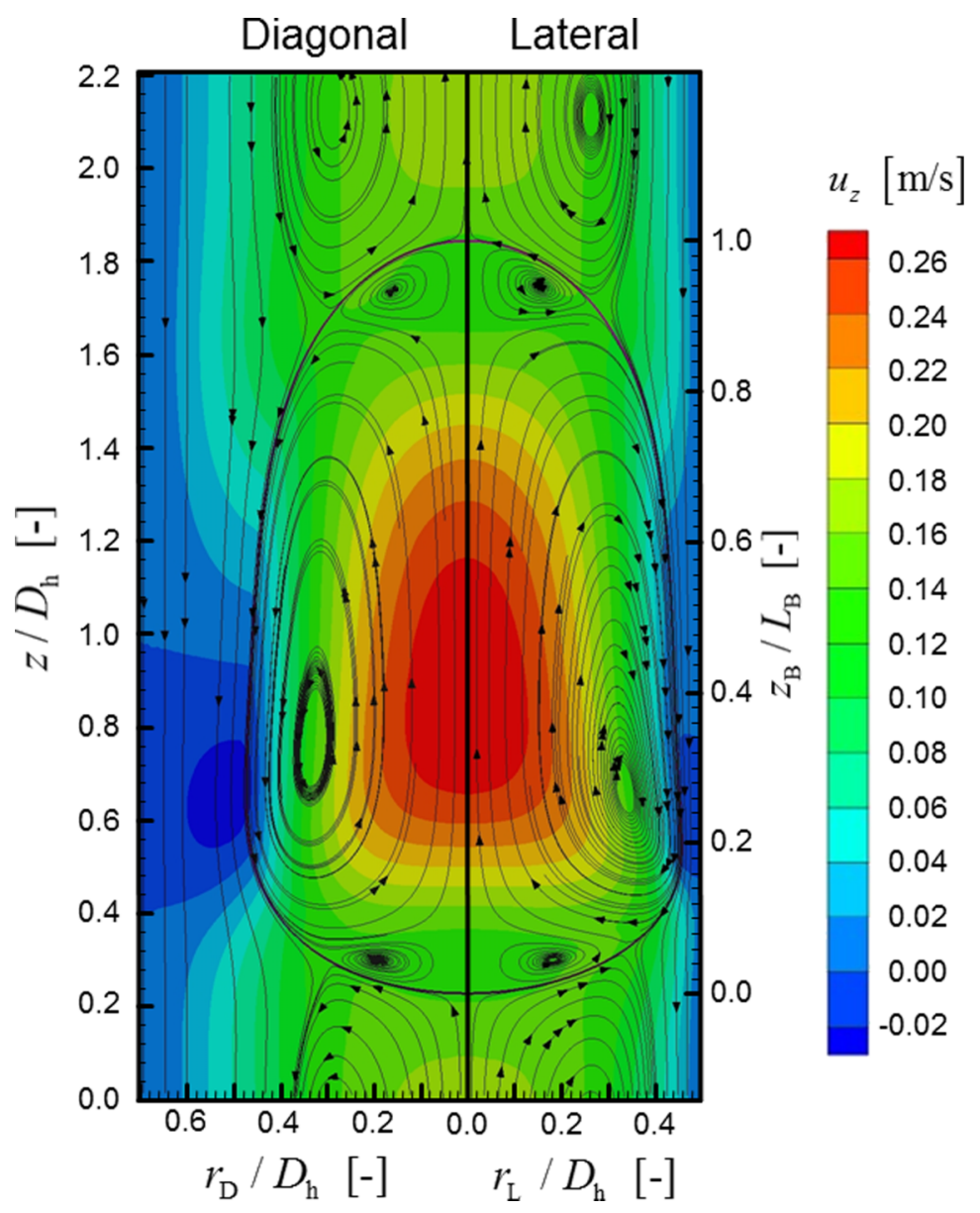

FIG. 5. Streamlines in the frame of reference moving with the bubble and vertical velocity in fixed frame of reference (color code) in a lateral and diagonal longitudinal cut (results of FS3D). 
reference (results of FS3D). The streamlines within the bubble indicate the presence of three toroidal vortices. The large central vortex in the body of the Taylor bubble exhibits high upward gas velocities close to the channel axis and low upward velocities close to the interface. This main central vortex drives two smaller toroidal vortices at the bubble front and bubble rear. The direction of rotation of both smaller vortices is opposite to that of the main vortex. The position of the center of the main vortex differs considerably in the lateral and diagonal cuts. This shows that the flow in the bubble is not axisymmetric but three-dimensional, especially in the lower part of the main vortex.

In the liquid slug, there is one main toroidal vortex which rotates in the same direction as the main vortex in the bubble. The occurrence of this recirculation pattern in the liquid phase was postulated by Taylor ${ }^{65}$ and was experimentally verified by Cox. ${ }^{66}$ Also visible is the dividing streamline, which separates the liquid slug in two regions, one with recirculating flow in the channel center and one with bypass flow close to the channel walls. ${ }^{17}$ The knowledge of the position of the dividing streamline is important for mechanistic modeling of mass transfer in Taylor flow. ${ }^{17,67}$ In the bypass region, the liquid velocity is low and one can identify in Fig. 5 local areas in the rear part of the liquid film where the liquid is flowing downwards.

Visualizations equivalent to Fig. 5 were also made for TURBIT-VOF and DROPS. Though the streamlines and contour lines are similar to those for FS3D displayed in Fig. 5, there are some notable differences in the location of the vortex centers and the radial position of the dividing streamline. These differences partly result from the different post-processing tools used by each code to obtain the streamlines. Furthermore, in the region near the front and rear menisci, the velocities are very small in the moving frame of reference (from which the streamlines are computed) so that the evaluation of streamlines becomes inaccurate. This may also explain why the streamlines in these regions are not closed in Fig. 5.

\section{Velocity field in the liquid slug}

For a detailed quantitative analysis of the velocity field in the liquid slug, local profiles of the axial and radial velocities are shown in Fig. 6 at three different axial positions, where also experimental data are available for comparison. Close to the front and rear menisci, the velocities show strong axial gradients. Therefore, the numerical profiles displayed in Fig. 6 were interpolated from the closest computational nodes to the specific respective axial position. The computed profiles of the axial velocity agree very well in the lateral near wall and corner regions with bypass flow, where they almost overlap for all three axial heights. Differences in the profiles of the axial velocity occur, however, in the channel center. These can partly be attributed to the different volumetric flow rates of the three codes. According to Table I, the volumetric flow rate of FS3D and DROPS is almost the same whereas the volumetric flow rate of TURBIT-VOF is about 3\% larger. The profiles of the axial velocity of FS3D and DROPS are virtually identical in Figs. 6(b) and 6(c), while close to the bubble rear a slight difference can be observed in the channel center, see Fig. 6(c). The higher axial velocity in the channel center computed by TURBIT-VOF (as compared to FS3D and DROPS) in Figs. 6(a) and 6(b) is consistent with its larger volumetric flow rate. Close to the bubble nose, the axial velocity in the channel center of TURBIT-VOF is, however, smaller as compared to the other codes, while the profile is slightly wider, see Fig. 6(c). In summary, the deviation of the computational and experimental results for the local centerline axial velocity is about $3 \%-5 \%$ for the three considered axial positions, while the deviation between the codes is less than $3 \%$.

The ratio between the maximum and mean axial velocity in the liquid slug is about 1.9. This value is lower than 2.096, which is the corresponding value of the fully developed laminar flow in a square channel. Thus, the liquid slug is not long enough for the velocity profile to become fully developed. This observation is consistent with experimental results from the literature. Cox ${ }^{66}$ reports a Poiseuille velocity distribution at a distance of 1.5 tube diameters ahead of the bubble, while Thulasidas et al. ${ }^{17}$ found that the Poiseuille profile within the liquid slug is fully developed for $L_{\mathrm{S}} / D_{\mathrm{h}} \geq 1.5$. In the present case, the ratio between slug length and hydraulic diameter is only $L_{\mathrm{S}} / D_{\mathrm{h}} \approx 0.6$.

The comparison of the computed lateral profiles of the axial velocity in the liquid slug with the experimental data yields a rather sophisticated picture. Close to the bubble rear the agreement is quite good, Fig. 6(a). The same holds for the magnitude of the axial velocity in the channel center close 

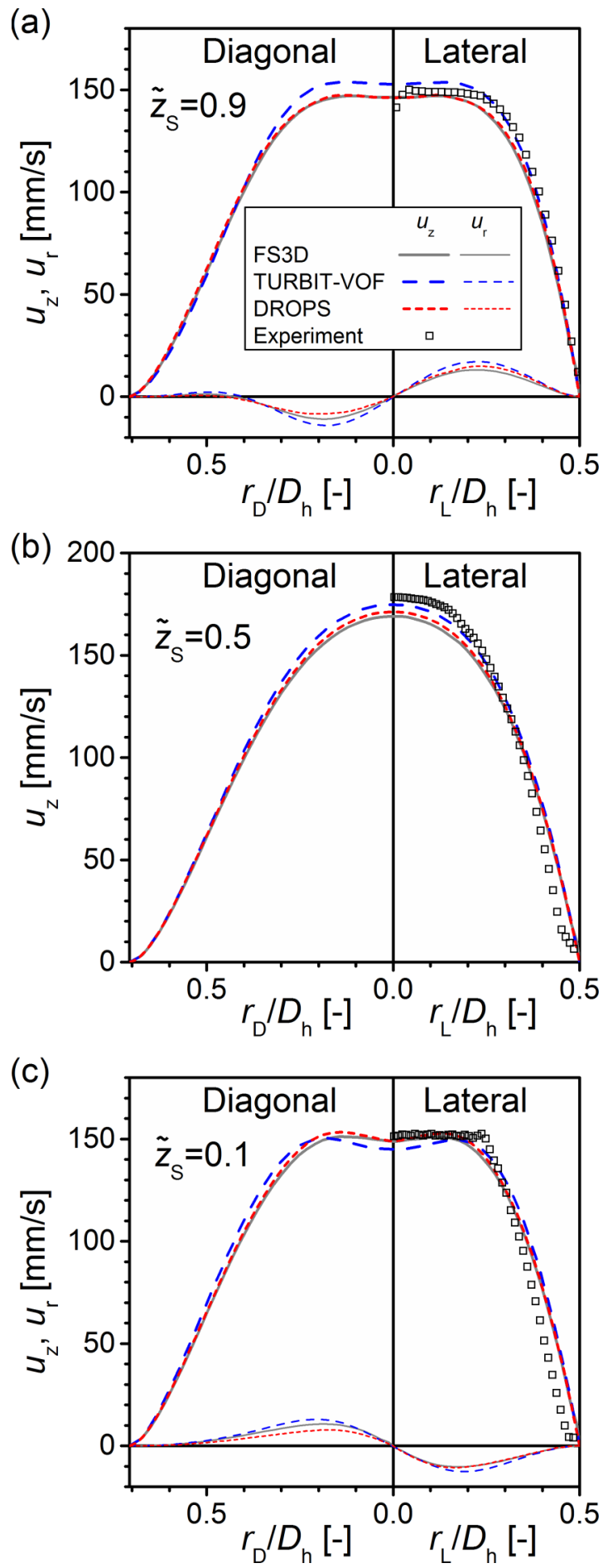

FIG. 6. Lateral and diagonal profiles of the axial and radial velocities in the liquid slug at different axial positions: (a) close to bubble rear $\left(\tilde{z}_{\mathrm{S}}=0.9\right)$, (b) in the middle of the liquid slug $\left(\tilde{z}_{\mathrm{S}}=0.5\right)$, and $(\mathrm{c})$ close to the bubble nose $\left(\tilde{z}_{\mathrm{S}}=0.1\right)$.

to the bubble nose, Fig. 6(c). In the middle of the liquid slug, the maximum axial velocity in the experiment is slightly higher than in the simulations, Fig. 6(b). A rather large deviation between the experimental data and the numerical profiles occurs close to the channel walls, both in the middle of the liquid slug, Fig. 6(b), and close to the bubble nose, Fig. 6(c). In both regions, the numerical results 
appear to be more reliable than the measured data. Uncertainties in the experimental data arise from the temperature dependence of the refractive index. Even minor changes in temperature may lead to optical refractive index mismatch at the channel wall. It is expected that a further development of the experimental setup (RIM and temperature control) will improve the accuracy of near wall velocity measurements.

Figs. 6(a) and 6(c) also show profiles of the radial liquid velocity in a lateral and diagonal cut. In the middle of the liquid slug, the radial velocity is virtually zero; the respective profiles are, therefore, not included in Fig. 6(b). The radial velocity profiles of all three codes are very similar but differ slightly in magnitude. Due to the recirculation pattern in the liquid slug (cf. Fig. 5), the radial liquid velocity is non-zero close to the front and rear menisci. Close to the bubble nose the liquid flows inward (i.e., toward the channel axis), while close to the bubble rear the liquid flow is outward. Figs. 6(a) and $6(\mathrm{c})$ show that at both locations, i.e., $\tilde{z}_{\mathrm{S}}=0.1$ and 0.9 , the magnitude of the radial velocity is only about $10 \%$ of the axial velocity.

\section{Velocity field in the bubble and the liquid film}

Figs. 7(a)-7(c) show similar velocity profiles as in Fig. 6 but for the bubble region. The respective axial positions correspond to $\tilde{z}_{\mathrm{B}}=0.25,0.5$, and 0.75 . Each velocity profile belongs partly to the liquid film and partly to the gas flow within the bubble. The exact radial positions of the interface in the lateral and diagonal cuts can be calculated for each code from the data given in Table II. In Figs. $7(a)-7(\mathrm{c})$, the average bubble diameter of the three codes at the respective axial position is marked by vertical dashed lines.

First, the velocity profiles inside the bubble are discussed. The results of FS3D and DROPS almost overlap for $\tilde{z}_{\mathrm{B}}=0.25$ but deviate by a few percent for $\tilde{z}_{\mathrm{B}}=0.5$ and 0.75 . TURBIT-VOF predicts a smaller magnitude of the axial velocity as compared to FS3D. The deviation in the magnitude of the computed centerline axial velocity between the different codes at the three considered axial positions is about $1.5 \%-5 \%$ and is the largest in the bubble nose. These differences are rather large, given the fact that the bubble velocity in all codes is very similar (the corresponding deviation is only $0.4 \%$ ). This can be attributed to the fact that the flow inside the bubble is more complex than that in the liquid slug.

For sufficiently large values of $r / D_{\mathrm{h}}$, the profiles in Figs. 7(a)-7(c) correspond to the velocity in the liquid film (say $r_{\mathrm{D} / \mathrm{L}} / D_{\mathrm{h}}>0.4-0.48$ depending on axial position). First, the results for the radial velocity are discussed qualitatively. In the diagonal cut, the magnitude of the radial velocity is very small close to the walls and the profile for radial velocity exhibits a horizontal tangent for all three axial positions. In the lateral cut, the magnitude of the radial velocity close to the wall is larger than in the diagonal cut and the horizontal tangent exists only for $\tilde{z}_{\mathrm{B}}=0.75$, see Fig. 7(a). The profiles for $\tilde{z}_{\mathrm{B}}=0.25$ and 0.5 show that liquid flows towards the wall. This is related to the axial profile of the bubble shape. Namely, the bubble diameter is increasing as $\tilde{z}_{\mathrm{B}}$ decreases (cf. Fig. 3), and the bubble pushes liquid toward the wall, where it is redistributed laterally toward the channel corners. This results in a draining of the lateral liquid film which will be discussed further in Section V.

For the axial velocity in the liquid film, experimental results are available for a comparison and included in Figs. 7(a)-7(c). The agreement between the three codes and the experimental results is rather good, both for the lateral and the diagonal cuts. This indicates that the slight differences in the computed bubble shape have only a minor effect on the flow in the liquid film. For $\tilde{z}_{\mathrm{B}}=0.75$ and 0.5 , an upward flow in the liquid film can be observed. For $\tilde{z}_{\mathrm{B}}=0.5$, the velocity profile in the liquid film is linear and of "Couette type" as discussed by Meyer et al. ${ }^{44}$ At $\tilde{z}_{\mathrm{B}}=0.25$, there is a local backflow of liquid in the film, both in the experiment as well as in the three computations. The magnitude of this backflow is much larger in the diagonal cut than in the lateral cut; see the inset in Fig. 7(c). This is consistent with the contour-plot of the vertical velocity shown in Fig. 5.

Since this paper considers co-current upward flow, one might conjecture that this local backflow is related to gravitational forces. However, this is not the case as is checked by a simulation with FS3D for the same value of $U_{\mathrm{B}}$ but without gravitation forces $(g=0)$. Also in this case, a local backflow in the rear part of the liquid film is found. 

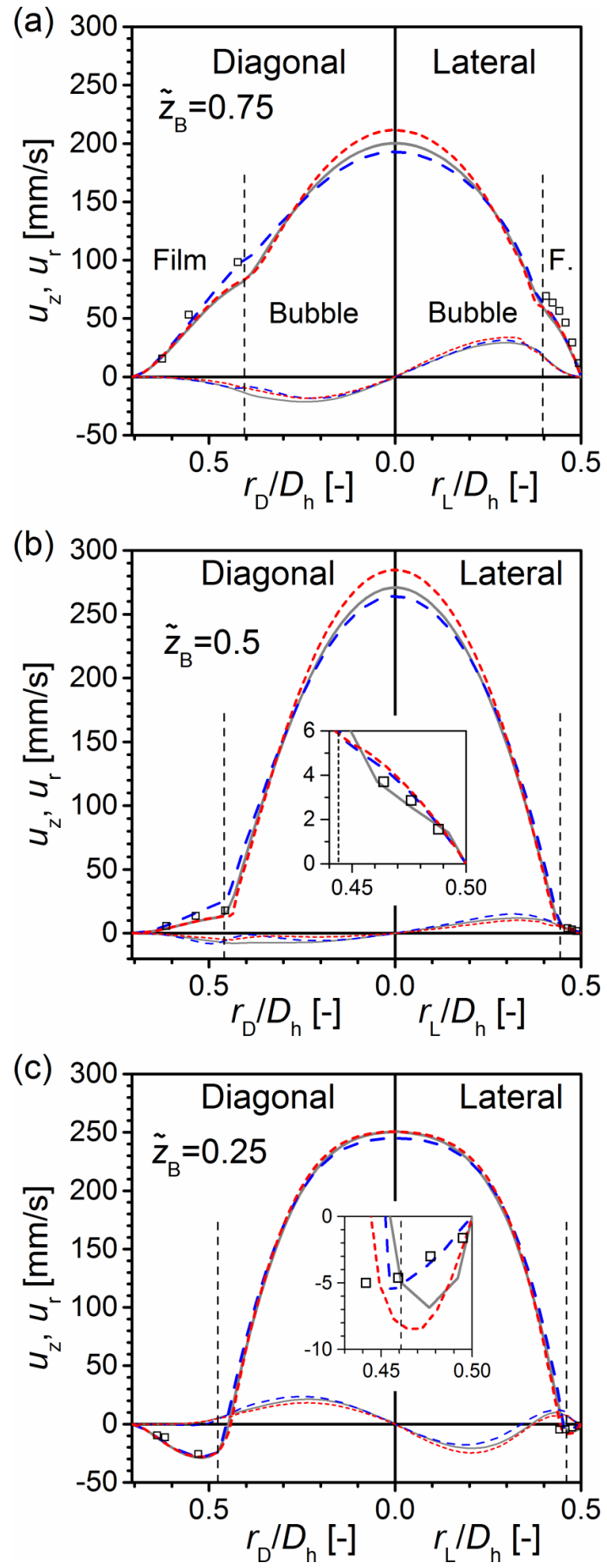

FIG. 7. Lateral and diagonal profiles of the axial and radial velocities at different axial positions in the bubble and the liquid film: (a) $\tilde{z}_{\mathrm{B}}=0.75$, (b) $\tilde{z}_{\mathrm{B}}=0.5$, (c) $\tilde{z}_{\mathrm{B}}=0.25$. The inset graphics in (b) and (c) show the lateral profiles of the axial velocity close to the wall. For the legend, see Fig. 6(a). The vertical dashed lines denote the average bubble diameter of the three codes at the respective axial position.

During the passage of a bubble, the local backflow causes a temporal reversal of the sign of the wall shear stress at a fixed position. This is of importance for heat and mass transfer applications with Taylor flow in various technical fields. An example in material science is the synthesis of particles with narrow size-distribution. ${ }^{68}$ In biology and medicine, lab-on-a-chip applications with Taylor 
flow are of interest for cell stimulus. ${ }^{15}$ Here, the shear stresses exerted on cells are of importance for the cell viability. ${ }^{69}$ In membranes for ultrafiltration, the alternating shear stresses of slug flow are of benefit for the cleaning and fouling control. ${ }^{70,71}$ Taha and $\mathrm{Cui}^{29,72}$ performed in this context numerical simulations with the volume-of-fluid method in the FLUENT code. They considered a single Taylor bubble in a vertical tube ${ }^{72}\left(20 \mathrm{~mm}\right.$ inner diameter) and in a square capillary ${ }^{29}\left(D_{h}=2 \mathrm{~mm}\right)$ and studied among others the wall shear stress distribution. For the square capillary, they found a reversal of the shear stress in lateral and diagonal directions both for upward and downward flows of the Taylor bubble. Moreover, the axial location of the wall shear stress reversal was found to be different in the lateral film and the channel corners. The authors did, however, not discuss the local velocity field in that region, nor did they give an explanation for the shear stress reversal.

\section{ANALYSIS OF LOCAL BACKFLOW IN THE REAR LIQUID FILM}

The most interesting result of Section IV is the occurrence of local backflow in the rear part of the liquid film. This section provides a physical explanation for this feature and gives criteria for its occurrence.

To investigate the phenomenon in more detail, advantage of the full simulation results is taken and the three-dimensional flow field is visualized at different locations. Fig. 8(a) shows the bubble shape, the isosurface of $u_{z}=-16 \mathrm{~mm} / \mathrm{s}$, and velocity vectors at the horizontal cross sections $\tilde{z}_{\mathrm{B}}=0.17,0.29$, and 0.52 (results of TURBIT-VOF). The velocity vectors indicate that the flow in the liquid film is upward at $\tilde{z}_{\mathrm{B}}=0.52$ and is downward at $\tilde{z}_{\mathrm{B}}=0.29$ and 0.17 . From the volume enclosed by the isosurface for $u_{z}=-0.16 \mathrm{~m} / \mathrm{s}$, it is evident that the backflow region in the liquid film is much larger in the channel corners than at the channel sides. For the two upper horizontal planes, the velocity in the lateral liquid film is quite small. In contrast, large downward velocities exist in the lowest plane close to the bubble rear, where the lateral film is the thinnest. The magnitude of the liquid velocity there is as high as the largest velocities in the corner region (cf. the local profiles of $u_{z}$ in Fig. 10 which will be discussed below).

The local backflow in the rear part of the liquid film is further illustrated in Figs. 9(a) and 9(b), where contour-plots of the vertical velocity $u_{z}$ are displayed for those two axial positions where the liquid film thickness is minimal in diagonal direction $\left(\tilde{z}_{\mathrm{B}}=0.27\right)$ and in lateral direction $\left(\tilde{z}_{\mathrm{B}}=0.17\right)$, respectively. Both figures confirm that the axial position where $u_{z}$ is minimal is different in both diagonal directions (cf. Fig. 8). A detailed inspection of the velocity fields displayed in Figs. 9(a) and 9(b) and in Fig. 8(a) indicates for $\tilde{z}_{\mathrm{B}}=0.27$ a draining liquid flow out of the lateral film into the channel
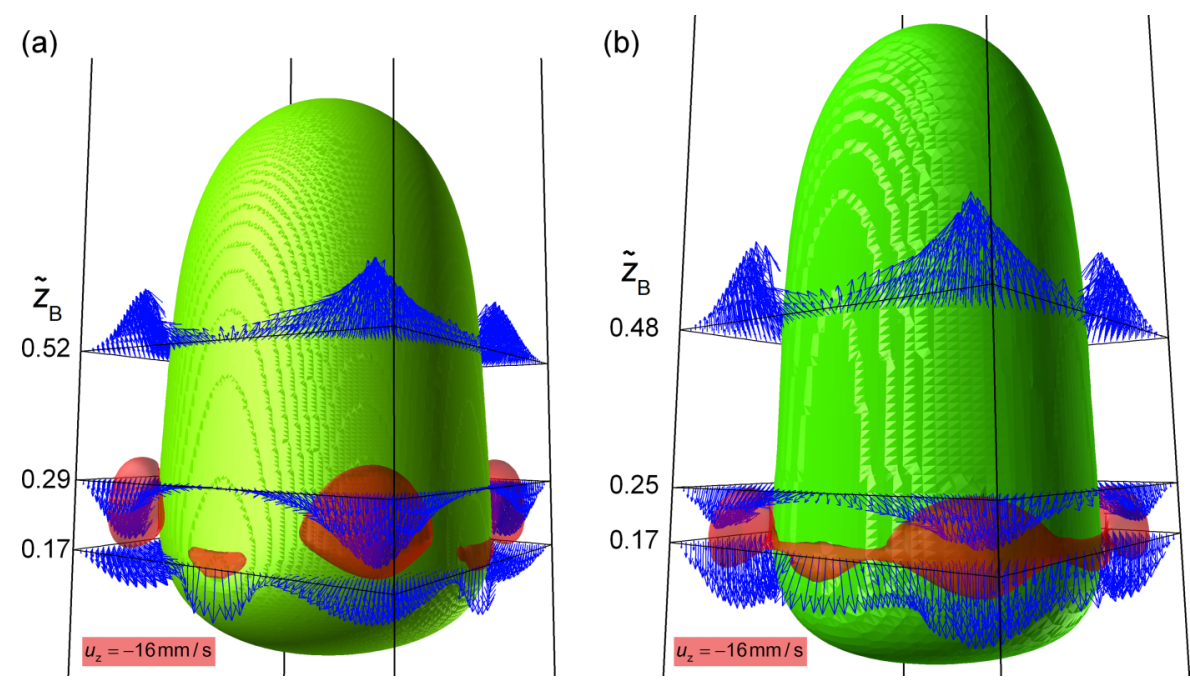

FIG. 8. Illustration of local backflow in the rear part of the liquid film (results of TURBIT-VOF). Visualization of the bubble shape, the isosurface of the vertical velocity $u_{z}=-16 \mathrm{~mm} / \mathrm{s}$, and the velocity field in three different horizontal cross sections. (a) Base case with $C a=0.1$ and (b) comparative case with $C a=0.23$. 
(a)

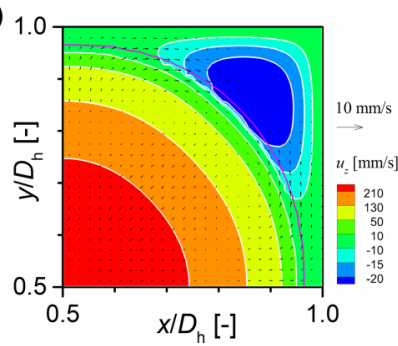

(b) 1

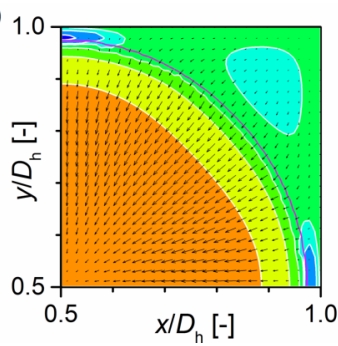

(c)

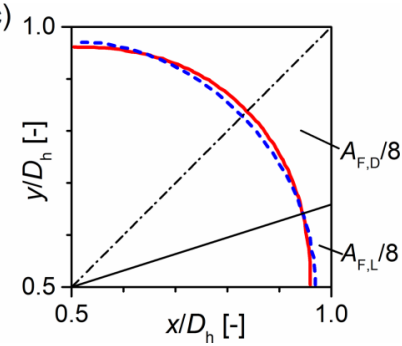

FIG. 9. (a) Contour plot of the vertical velocity $u_{z}$ in one quarter of the cross section at position $\tilde{z}_{\mathrm{B}}=0.27$ where the diagonal liquid film is the thinnest (the red line indicates the interface, every 2nd velocity vector is displayed in $x$ - and $y$-directions, results of TURBIT-VOF). (b) Same but for position $\tilde{z}_{\mathrm{B}}=0.17$ where the lateral film is the thinnest. (c) Interface profiles in cross section $\tilde{z}_{\mathrm{B}}=0.27$ (red solid line) and $\tilde{z}_{\mathrm{B}}=0.17$ (blue dashed line) and illustration of the lateral and diagonal liquid film areas.

corners, in agreement with the numerical study of Hazel and Heil. ${ }^{64}$ At $\tilde{z}_{\mathrm{B}}=0.17$ close to the rear meniscus, the flow direction in the liquid film is opposite, i.e., from the corner regions towards the lateral film.

A useful concept in Taylor flow is a macroscopic material balance of the liquid flow. By considering a cross section where gas and liquid are present and a cross section through the liquid slug, a relation between the average liquid velocity and the bubble velocity can be established. ${ }^{6,73}$ In a frame of reference moving with the bubble, this mass balance leads — under the assumptions of quasi-steady state and incompressible phases - to the relation

$$
\left(J-U_{\mathrm{B}}\right) A=\left[U_{\mathrm{F}}\left(z_{\mathrm{B}}\right)-U_{\mathrm{B}}\right] A_{\mathrm{F}}\left(z_{\mathrm{B}}\right) .
$$

Here, $U_{\mathrm{F}}\left(z_{\mathrm{B}}\right)$ is the average axial velocity in the liquid film in a cross section at a certain axial position $z_{\mathrm{B}}, J$ is the total superficial velocity, $A$ is the area of the channel cross section, and $A_{\mathrm{F}}\left(z_{\mathrm{B}}\right)=$ $A-A_{\mathrm{B}}\left(z_{\mathrm{B}}\right)$ is the cross-sectional area of the liquid phase. Abiev ${ }^{74}$ pointed out that Eq. (4) implies that $U_{\mathrm{F}}$ becomes negative for $A_{\mathrm{B}}\left(z_{\mathrm{B}}\right) / A>J / U_{\mathrm{B}}$ which corresponds to a local flow reversal in the liquid film.

For an axisymmetric bubble in a square channel, it is $A_{\mathrm{B}} / A=\pi D_{\mathrm{B}}^{2} / 4 D_{\mathrm{h}}^{2}$ and Eq. (4) yields

$$
U_{\mathrm{F}}\left(z_{\mathrm{B}}\right)=\frac{J-U_{\mathrm{B}} \frac{\pi}{4} \frac{D_{\mathrm{B}}^{2}\left(z_{\mathrm{B}}\right)}{D_{\mathrm{h}}}}{1-\frac{\pi}{4} \frac{D_{\mathrm{B}}^{2}\left(z_{\mathrm{B}}\right)}{D_{\mathrm{h}}^{2}}} .
$$

This relation can be used to compute $U_{\mathrm{F}}\left(z_{\mathrm{B}}\right)$ from the axial profile of the bubble diameter $D_{\mathrm{B}}\left(z_{\mathrm{B}}\right)$. In the present case, the bubble is not axisymmetric and has slightly different dimensions in lateral and diagonal directions (cf. Fig. 3 and Table II). Here, the main interest is in the local axial velocity $u_{z}$ in the lateral and diagonal liquid film regions and not in the mean axial velocity $U_{\mathrm{F}}$. Nevertheless, it is interesting to evaluate Eq. (5) under the hypothetical assumption that the bubble is axisymmetric, once with the lateral bubble diameter profile $D_{\mathrm{B}, \mathrm{L}}\left(z_{\mathrm{B}}\right)$ and once with the diagonal bubble diameter profile $D_{\mathrm{B}, \mathrm{D}}\left(z_{\mathrm{B}}\right)$ from Fig. 3. Given the similar interface profiles for the three codes, the respective curves for $U_{\mathrm{F}}\left(z_{\mathrm{B}}\right)$ are very similar, too. Therefore, in Fig. 10, only the curves computed from the interface profiles of TURBIT-VOF are shown. The figure shows that for both the bubble profile in lateral and in diagonal directions, there is a region where the predicted value of $U_{\mathrm{F}}$ is negative which indicates backflow. In lateral direction, the predicted axial region with backflow is smaller and located at a lower height as compared to the diagonal direction. These results are consistent with the contour-plot of the velocity field of $u_{z}$ in Fig. 5 (from FS3D) and the isosurface for $u_{z}=-0.16 \mathrm{~m} / \mathrm{s}$ in Fig. 8(a) (from TURBIT-VOF). Both figures show that the axial region where $u_{z}$ is negative differs in the lateral and diagonal cuts.

Also shown in Fig. 10 are local velocity profiles of $u_{z}$ in the middle of the lateral and diagonal liquid films (results of TURBIT-VOF). The profiles of $u_{z}$ in the lateral and diagonal liquid films at other radial positions (not shown here) are qualitatively very similar to those displayed in Fig. 10, but differ in magnitude. Comparing the profiles of $U_{\mathrm{F}}$ and $u_{z}$ in Fig. 10 shows that Eq. (5) predicts the 


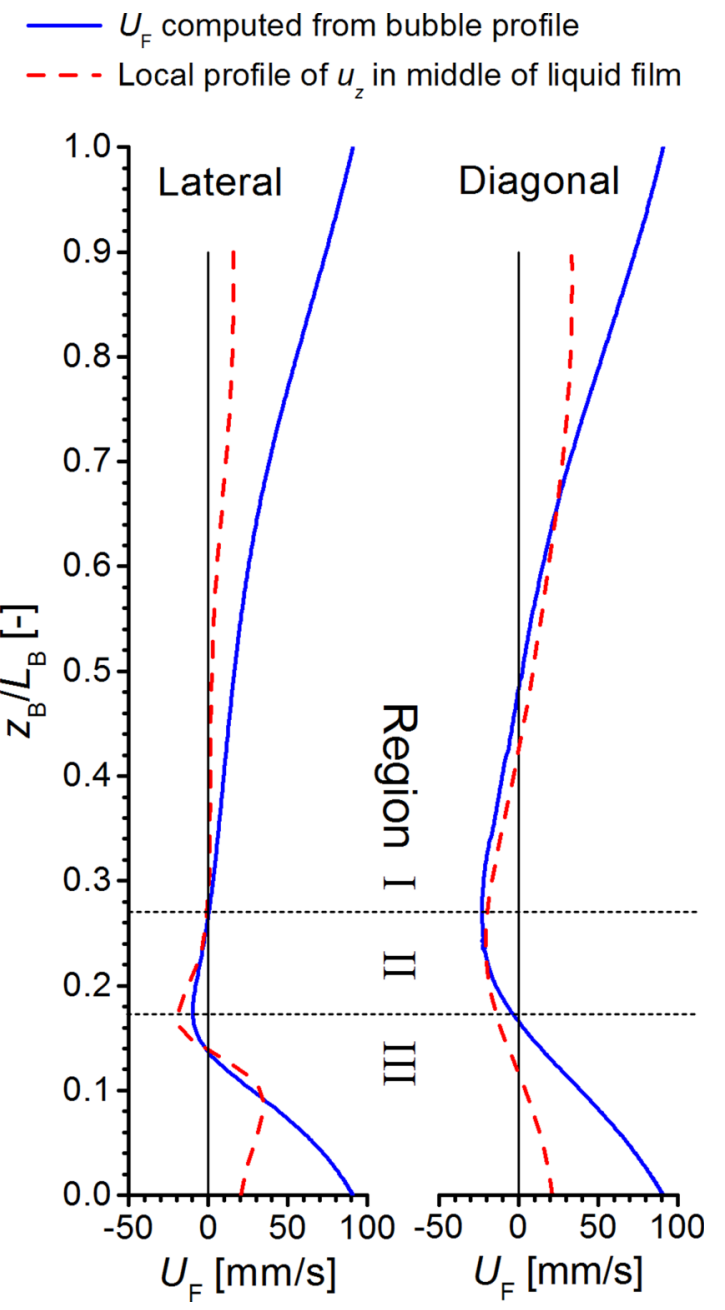

FIG. 10. Vertical profile of the mean axial velocity in the liquid film, $U_{\mathrm{F}}$, as computed by Eq. (5) from the lateral and diagonal interface profiles, and vertical profile of the local axial velocity $\boldsymbol{u}_{z}$ in the lateral and diagonal liquid films at positions $\tilde{r}_{\mathrm{L}}=0.484$ and $\tilde{r}_{\mathrm{D}}=0.592$, respectively (results from TURBIT-VOF). The two dashed horizontal lines at $\tilde{z}_{\mathrm{B}}=0.17$ and 0.27 indicate the positions where the liquid film thickness is the thinnest in lateral and diagonal directions, respectively.

region with backflow in lateral direction quite well. For the diagonal liquid film, however, the local velocity profile indicates that the region with backflow is actually located somewhat closer to the rear meniscus than is predicted by Eq. (5). The reason for this discrepancy is probably the drainage of the lateral liquid film and the associated movement of liquid toward the corners which is visible in Fig. 9(b) and which is not taken into account by the above simplified analysis.

Next it is investigated why the locations where $U_{\mathrm{F}}$ is minimal differ so much in lateral and diagonal directions. By taking the derivative of Eq. (4) with respect to $z_{\mathrm{B}}$, one obtains

$$
\frac{\partial U_{\mathrm{F}}\left(z_{\mathrm{B}}\right)}{\partial z_{\mathrm{B}}}=\underbrace{\frac{U_{\mathrm{B}}-U_{\mathrm{F}}\left(z_{\mathrm{B}}\right)}{A_{\mathrm{F}}\left(z_{\mathrm{B}}\right)}}_{>0} \frac{\partial A_{\mathrm{F}}\left(z_{\mathrm{B}}\right)}{\partial z_{\mathrm{B}}} .
$$

From Eq. (6) it follows that the axial position where $U_{\mathrm{F}}$ is minimal is identical to the position where $A_{\mathrm{F}}$ is minimal. For an axisymmetric bubble in a circular pipe, the thickness of the liquid film is uniform in azimuthal direction so that the axial position where $U_{\mathrm{F}}$ is minimal is azimuthally unique. In a square capillary, the thickness of the liquid film is not uniform within an axial cross section but varies in azimuthal direction. The liquid film is the thinnest in lateral direction and the thickest in diagonal direction. To account for this, the area of the liquid film is represented as a sum of two parts, i.e., 
$A_{\mathrm{F}}=A_{\mathrm{F}, \mathrm{L}}+A_{\mathrm{F}, \mathrm{D}}$. Here, $A_{\mathrm{F}, \mathrm{L}}\left(z_{\mathrm{B}}\right)$ is the area of the lateral liquid film and $A_{\mathrm{F}, \mathrm{D}}\left(z_{\mathrm{B}}\right)$ is the area of the film in the corner. The experiments by Fries et al. ${ }^{20}$ for capillary numbers in the range 0.0002-0.01 show that the corners can contribute with about $70 \%$ to the total film area. An exact definition of both areas is, however, difficult. Fig. 9(c) shows the interface profiles in one quarter of the channel cross section at the two axial positions where the thickness of the liquid film is minimal in lateral and diagonal directions, respectively. In the present case, both curves intersect at an angle of $18.5^{\circ}$ and $A_{\mathrm{F}, \mathrm{L}}$ and $A_{\mathrm{F}, \mathrm{D}}$ are defined accordingly.

By splitting the liquid volumetric flux in two parts (i.e., through area $A_{\mathrm{F}, \mathrm{L}}$ or $A_{\mathrm{F}, \mathrm{D}}$ ), the liquid mass balance from Eq. (4) becomes

$$
\left(J-U_{\mathrm{B}}\right) A=\left[U_{\mathrm{F}, \mathrm{L}}\left(z_{\mathrm{B}}\right)-U_{\mathrm{B}}\right] A_{\mathrm{F}, \mathrm{L}}\left(z_{\mathrm{B}}\right)+\left[U_{\mathrm{F}, \mathrm{D}}\left(z_{\mathrm{B}}\right)-U_{\mathrm{B}}\right] A_{\mathrm{F}, \mathrm{D}}\left(z_{\mathrm{B}}\right) .
$$

Taking the derivative of Eq. (7) with respect to $z_{\mathrm{B}}$ gives

$$
\underbrace{A_{\mathrm{F}, \mathrm{L}}}_{>0} \frac{\partial U_{\mathrm{F}, \mathrm{L}}}{\partial z_{\mathrm{B}}}+\underbrace{A_{\mathrm{F}, \mathrm{D}}}_{>0} \frac{\partial U_{\mathrm{F}, \mathrm{D}}}{\partial z_{\mathrm{B}}}=\underbrace{\left[U_{\mathrm{B}}-U_{\mathrm{F}, \mathrm{D}}\left(z_{\mathrm{B}}\right)\right]}_{>0} \frac{\partial A_{\mathrm{F}, \mathrm{D}}}{\partial z_{\mathrm{B}}}+\underbrace{\left[U_{\mathrm{B}}-U_{\mathrm{F}, \mathrm{L}}\left(z_{\mathrm{B}}\right)\right]}_{>0} \frac{\partial A_{\mathrm{F}, \mathrm{L}}}{\partial z_{\mathrm{B}}} .
$$

To discuss the implications of Eq. (8), it is useful to consider again Fig. 10 where three axial regions in the liquid film can be distinguished. In region $\mathrm{I}$ at the bubble front it is $\partial A_{\mathrm{F}, \mathrm{D}} / \partial z_{\mathrm{B}}>0$ and $\partial A_{\mathrm{F}, \mathrm{L}} / \partial z_{\mathrm{B}}>0$ (cf. the interface profiles in Fig. 3) so that the right-hand side of Eq. (8) is positive. For this region, one may expect from Eq. (8) $\partial U_{\mathrm{F}, \mathrm{L}} / \partial z_{\mathrm{B}}>0$ and $\partial U_{\mathrm{F}, \mathrm{D}} / \partial z_{\mathrm{B}}>0$. In the liquid film near the bubble rear (region III) it is $\partial A_{\mathrm{F}, \mathrm{L}} / \partial z_{\mathrm{B}}<0$ and $\partial A_{\mathrm{F}, \mathrm{D}} / \partial z_{\mathrm{B}}<0$ so that the right-hand side of Eq. (8) is negative. In this region, one may expect from Eq. (8) $\partial U_{\mathrm{F}, \mathrm{L}} / \partial z_{\mathrm{B}}<0$ and $\partial U_{\mathrm{F}, \mathrm{D}} / \partial z_{\mathrm{B}}<0$. Of special interest here is region II where $\partial A_{\mathrm{F}, \mathrm{L}} / \partial z_{\mathrm{B}} \geq 0$ and $\partial A_{\mathrm{F}, \mathrm{D}} / \partial z_{\mathrm{B}} \leq 0$. The magnitude of $U_{\mathrm{F}, L}$ and $U_{\mathrm{F}, D}$ in this region is about one order smaller than that of $U_{\mathrm{B}}$. Therefore, in the right-hand side of Eq. (8), the approximations $U_{\mathrm{B}}-U_{\mathrm{F}, \mathrm{D}}\left(z_{\mathrm{B}}\right) \approx U_{\mathrm{B}}$ and $U_{\mathrm{B}}-U_{\mathrm{F}, \mathrm{L}}\left(z_{\mathrm{B}}\right) \approx U_{\mathrm{B}}$ can be introduced which yields

$$
A_{\mathrm{F}, \mathrm{D}} \frac{\partial U_{\mathrm{F}, \mathrm{D}}}{\partial z_{\mathrm{B}}} \approx U_{\mathrm{B}} \frac{\partial A_{\mathrm{F}}}{\partial z_{\mathrm{B}}}-A_{\mathrm{F}, \mathrm{L}} \frac{\partial U_{\mathrm{F}, \mathrm{L}}}{\partial z_{\mathrm{B}}} .
$$

This equation establishes a relation between the axial derivatives of the mean velocity in the lateral and diagonal liquid films. For the present case, the local profile of $u_{z}$ in the lateral film in Fig. 10 indicates that in the entire region II it is $\partial U_{\mathrm{F}, \mathrm{L}} / \partial z_{\mathrm{B}} \geq 0$. Somewhere within region II, the film area $A_{\mathrm{F}}\left(z_{\mathrm{B}}\right)$ takes its minimum value. Thus, within region II there are two sub-regions, one where $\partial A_{\mathrm{F}} / \partial z_{\mathrm{B}}<0$ (close to region III) and one where $\partial A_{\mathrm{F}} / \partial z_{\mathrm{B}}>0$ (close to region I). In the former sub-region, the right-hand side of Eq. (9) is negative; thus it follows $\partial U_{\mathrm{F}, \mathrm{D}} / \partial z_{\mathrm{B}}<0$ and the vertical derivatives of the mean velocity in the liquid film in lateral and diagonal directions have opposite signs. This behavior is clearly reflected by the local profiles of $u_{z}$ in the lateral and diagonal films in Fig. 10. In the latter sub-region of region II, the right-hand side of Eq. (9) may be positive or negative and a clear statement about the sign of the derivate $\partial U_{\mathrm{F}, \mathrm{D}} / \partial z_{\mathrm{B}}$ is not possible.

In summary, it follows by a mass balance of the liquid flow in the corner and at the channel sides that it is the axial change of $A_{\mathrm{F}, \mathrm{L}}$ and $A_{\mathrm{F}, \mathrm{D}}$ displayed in Fig. 9(c) which gives rise to region II in Fig. 10 with different axial locations where $u_{z}$ is minimal (and negative) in lateral and diagonal directions. The axial change of $A_{\mathrm{F}, \mathrm{L}}$ and $A_{\mathrm{F}, \mathrm{D}}$ in the rear part of the liquid film is related to the axial variation of the bubble aspect ratio which is caused by the non-uniform azimuthal pressure distribution within a cross section (cf. Section IV A and Fig. 4).

An implication of the conjecture from the last paragraph is that the size of region II should diminish or even disappears for Taylor bubbles with higher capillary number since these are axisymmetric. This is tested by the comparative simulation, where $C a=0.23$ (cf. last paragraph in Sec. III C). For this comparative case, the ratio between the maximum lateral and maximum diagonal bubble radii is 0.99 as compared to 0.98 for the base case. Furthermore, the relative difference between the axial positions where both maxima occur (i.e., the length of region II) takes a value of $\Delta z_{\mathrm{B}} / D_{\mathrm{h}}=0.080$ while it was 0.175 for the base case. Fig. 8(b) shows for the comparative case a visualization equivalent to the one displayed in Fig. 8(a) for the base case (where $C a=0.1$ ). The maximum local downward velocity occurs within the isosurface for $u_{z}=-16 \mathrm{~mm} / \mathrm{s}$. Both in Figs. 8(a) and 8(b), the isosurface volume is larger in the corners than in the lateral film. However, in contrast to Fig. 8(a), the isosurface 
forms in Fig. 8(b) a connected tube around the bubble and the axial position of the isosurface is about the same in the lateral and diagonal films. The downward velocity vectors in Fig. 8(a) increase in magnitude from the plane at $\tilde{z}_{B}=0.29$ to the one at 0.17 in the lateral film, but decrease in the diagonal film. In Fig. 8(b), the downward velocity vectors increase in magnitude from the plane at $\tilde{z}_{B}=0.25$ to the one at 0.17 , both in the lateral and the diagonal films. Thus, there is no region visible where the axial gradients of the axial velocity show opposite signs in the lateral and diagonal films. This and the significant decrease of the size of region II as the capillary number is increased from $C a=0.1$ to 0.23 confirm the conjecture from the previous paragraph. Finally, it is worth to remark that even for $C a=0.23$ the Taylor bubble is still locally non-axisymmetric. Similar to the base case, the ratio between the local lateral and diagonal bubble diameter is larger than unity close to the rear meniscus (with a maximum of about 1.04), while it is smaller than unity at the bubble body and nose (with a minimum value of about 0.97 ). Thus, as compared to the base case, this local deviation from rotational symmetry has decreases as well, as did the global deviation from rotational symmetry (measured by the ratio of the maximum lateral to maximum diagonal bubble radius).

For practical applications with Taylor flow it may be useful to estimate beforehand whether a local backflow in the liquid film is to be expected or not. From Eq. (4), it follows that a sufficient condition for local backflow is

$$
\frac{A_{\mathrm{B}, \max }}{A} \cdot \frac{U_{\mathrm{B}}}{J}>1,
$$

where $A_{\mathrm{B}, \max }$ is the maximum value of the cross-sectional area of the bubble. The first ratio on the left-hand side of Eq. (10) is always less than unity, whereas the second ratio is always larger than unity. Both ratios depend on the capillary number. Liu et al. ${ }^{19}$ performed experiments in capillaries with circular and square cross section for various fluids and derived a correlation for $U_{\mathrm{B}} / J$. For circular channels, the correlation of $\mathrm{Abiev}^{67}$ for $U_{\mathrm{B}} / J$ (which is valid for a wider range of capillary numbers) can be used as well while the ratio $A_{\mathrm{B}, \max } / A$ can be estimated from the correlation of Aussillous and Quere ${ }^{75}$ for the liquid film thickness. This allows to test the inequality in Eq. (10) for circular channels.

For square channels, the determination of $A_{\mathrm{B}, \max } / A$ is more subtle and one has to distinguish between globally axisymmetric bubbles and globally non-axisymmetric bubbles. According to Kreutzer et al. ${ }^{11}$ the transition between both regimes occurs at $C a \approx 0.04$. The latter authors provide a correlation for the bubble diameter in the axisymmetric regime. For the non-axisymmetric regime, they provide correlations for the bubble diameter in lateral and diagonal directions. For the non-axisymmetric case, these correlations allow in combination with the geometrical considerations reported in the appendix of Kolb and Cerro ${ }^{63}$ to estimate $A_{\mathrm{B}, \max } / A$ and thus to test the criterion in Eq. (10). Alternatively, one can use the correlation for $A_{\mathrm{B}}$ given by Haase and Bauer ${ }^{23}$ which is based on the assumption $D_{\mathrm{B}, \text { max:L }} / D_{\mathrm{h}}=0.95$. In the present simulations, the latter ratio has a value of about 0.93 . Finally, it is noted that in the present study and in our previous one ${ }^{37}$ the bubble is already slightly globally non-axisymmetric for $C a \approx 0.1$. If the Taylor bubble in a square channel is not globally axisymmetric, then the local backflow in the lateral and diagonal film will occur in different axial regions as shown above.

\section{CONCLUSIONS}

This paper presents a coordinated numerical and experimental study on local flow phenomena in co-current upward laminar Taylor flow through a small square channel. In the computations with three academic computer codes, the bubble volume and velocity as well as the size of the Taylor flow unit cell are chosen in accordance with the experiments (with values of the capillary and Reynolds number of 0.1 and 7 , respectively). For these parameters, the local numerical results for the bubble radius agree with a deviation of about $0.6 \%$. For the local centerline axial velocity, the results agree with a deviation of less than $3 \%$ in the liquid slug and of less than about $5 \%$ for the flow within the bubble.

Parameters which are sensitive with respect to the numerical method are the minimum thickness of the lateral liquid film and the axial position where the minimum thickness occurs. While the agreement regarding the bubble shape and the velocity field in the liquid slug and in the liquid film is reasonably good, the differences concerning the flow in the bubble are larger. From this, one 
can conclude that a reasonable good agreement for the bubble shape and bubble velocity does not automatically imply a good agreement in the local velocity field within the bubble. The reasons for the rather large discrepancies concerning the flow within the bubble are yet unclear. Uncovering them requires detailed experimental data on the velocity field within the Taylor bubble as well as further computational studies and benchmark data on the local velocity and pressure fields within the Taylor bubble.

The present experimental and computational results consistently show a local backflow region in the rear part of the liquid film in the fixed frame of reference. This is associated with a temporal reversal of the wall shear stress during the passage of a Taylor bubble. This local backflow occurs at different axial positions in the lateral film and in the corner region. By a detailed analysis of the full three-dimensional simulation results, it is shown that this novel phenomenon has its origin in a slight deviation of the Taylor bubble shape from rotational symmetry. Of special importance is the crossover of the local bubble aspect ratio (defined as the local ratio between the bubble diameter in diagonal and lateral directions at a certain axial position) from values larger than unity at the bubble nose and bubble body to values smaller than unity at the bubble rear.

Our study considered two specific values of the capillary number. Future studies may investigate the existence and size of this local backflow region for capillary and Reynolds numbers varying in a wider range. So far, approximate criteria are given for the conditions under which the shear stress reversal occurs in circular and square channels. These and the detailed understanding of the underlying physical mechanism shall be useful for some technical applications with gas-liquid Taylor flow (e.g., material synthesis). Finally, it is likely that similar phenomena will also occur in channels with other polygonal cross section, such as trapezoidal or equilateral triangular channels.

\section{ACKNOWLEDGMENTS}

The authors wish to thank the German Research Foundation (DFG) for financial support within the Priority Programme "Transport Processes at Fluidic Interfaces" (SPP 1506) with the Project Nos. BO 1879/9-1, RE 1461/2-1, SCHL 617/7-1, and WO 1682/1-1.

${ }^{1}$ S. Osher and R. P. Fedkiw, Level Set Methods and Dynamic Implicit Surfaces (Springer, Berlin, London, 2003).

${ }^{2}$ G. Tryggvason, R. Scardovelli, and S. Zaleski, Direct Numerical Simulations of Gas-Liquid Multiphase Flows (Cambridge University Press, Cambridge, 2011).

${ }^{3}$ A. Prosperetti and G. Tryggvason, Computational Methods for Multiphase Flow (Cambridge University Press, Cambridge, 2007).

${ }^{4}$ S. Groß and A. Reusken, Numerical Methods for Two-Phase Incompressible Flows (Springer, Heidelberg, 2011).

${ }^{5} \mathrm{M}$. Wörner, "Numerical modeling of multiphase flows in microfluidics and micro process engineering: A review of methods and applications," Microfluid. Nanofluid. 12, 841 (2012).

${ }^{6}$ M. Suo and P. Griffith, "Two-phase flow in capillary tubes," J. Basic Eng. 86, 576 (1964).

${ }^{7}$ D. T. Dumitrescu, "Strömung an einer luftblase im senkrechten Rohr," Z. Angew. Math. Mech. 23, 139 (1943).

${ }^{8}$ R. M. Davies and G. Taylor, "The mechanics of large bubbles rising through extended liquids and through liquids in tubes," Proc. R. Soc. A 200, 375 (1950).

${ }^{9}$ K. Jähnisch, M. Baerns, V. Hessel, W. Ehrfeld, V. Haverkamp, H. Löwe, C. Wille, and A. Guber, "Direct fluorination of toluene using elemental fluorine in gas/liquid microreactors," J. Fluorine Chem. 105, 117 (2000).

${ }^{10}$ M. Al-Rawashdeh, J. Zalucky, C. Muller, T. A. Nijhuis, V. Hessel, and J. C. Schouten, "Phenylacetylene hydrogenation over $\left[\mathrm{Rh}(\mathrm{NBD})\left(\mathrm{PPh}_{3}\right)_{2}\right] \mathrm{BF}_{4}$ catalyst in a numbered-up microchannels reactor," Ind. Eng. Chem. Res. 52, 11516 (2013).

${ }^{11}$ M. T. Kreutzer, F. Kapteijn, J. A. Moulijn, and J. J. Heiszwolf, "Multiphase monolith reactors: Chemical reaction engineering of segmented flow in microchannels," Chem. Eng. Sci. 60, 5895 (2005).

12 A. Günther and K. F. Jensen, "Multiphase microfluidics: From flow characteristics to chemical and materials synthesis," Lab Chip 6, 1487 (2006).

${ }^{13}$ J. I. Park, A. Saffari, S. Kumar, A. Günther, and E. Kumacheva, "Microfluidic synthesis of polymer and inorganic particulate materials," Annu. Rev. Mater. Res. 40, 415 (2010).

${ }^{14} \mathrm{~J}$. M. Köhler, S. N. Li, and A. Knauer, "Why is micro segmented flow particularly promising for the synthesis of nanomaterials?," Chem. Eng. Technol. 36, 887 (2013).

${ }^{15}$ J. El-Ali, S. Gaudet, A. Günther, P. K. Sorger, and K. F. Jensen, "Cell stimulus and lysis in a microfluidic device with segmented gas-liquid flow," Anal. Chem. 77, 3629 (2005).

${ }^{16}$ T. C. Thulasidas, M. A. Abraham, and R. L. Cerro, "Bubble-train flow in capillaries of circular and square cross-section," Chem. Eng. Sci. 50, 183 (1995).

${ }^{17}$ T. C. Thulasidas, M. A. Abraham, and R. L. Cerro, "Flow patterns in liquid slugs during bubble-train flow inside capillaries," Chem. Eng. Sci. 52, 2947 (1997).

${ }^{18}$ T. Cubaud and C. M. Ho, "Transport of bubbles in square microchannels," Phys. Fluids 16, 4575 (2004). 
${ }^{19}$ H. Liu, C. O. Vandu, and R. Krishna, "Hydrodynamics of Taylor flow in vertical capillaries: Flow regimes, bubble rise velocity, liquid slug length, and pressure drop," Ind. Eng. Chem. Res. 44, 4884 (2005).

${ }^{20}$ D. M. Fries, F. Trachsel, and P. R. von Rohr, "Segmented gas-liquid flow characterization in rectangular microchannels," Int. J. Multiphase Flow 34, 1108 (2008).

${ }^{21}$ M. J. F. Warnier, E. Rebrov, M. H. J. M. De Croon, V. Hessel, and J. C. Schouten, "Gas hold-up and liquid film thickness in Taylor flow in rectangular microchannels," Chem. Eng. J. 135, S153 (2008).

${ }^{22}$ Y. Han and N. Shikazono, "Measurement of liquid film thickness in micro square channel," Int. J. Multiphase Flow 35, 896 (2009).

${ }^{23} \mathrm{~S}$. Haase and T. Bauer, "New method for simultaneous measurement of hydrodynamics and reaction rates in a mini-channel with Taylor flow," Chem. Eng. J. 65, 176-177 (2011).

${ }^{24}$ M. Roudet, K. Loubiere, C. Gourdon, and M. Cabassud, "Hydrodynamic and mass transfer in inertial gas-liquid flow regimes through straight and meandering millimetric square channels," Chem. Eng. Sci. 66, 2974 (2011).

${ }^{25}$ P. Zaloha, J. Kristal, V. Jiricny, N. Volkel, C. Xuereb, and J. Aubin, "Characteristics of liquid slugs in gas-liquid Taylor flow in microchannels," Chem. Eng. Sci. 68, 640 (2012).

${ }^{26}$ T. Abadie, J. Aubin, D. Legendre, and C. Xuereb, "Hydrodynamics of gas-liquid Taylor flow in rectangular microchannels," Microfluid. Nanofluid. 12, 355 (2012).

${ }^{27}$ S. Boden, T. dos Santos Rolo, T. Baumbach, and U. Hampel, "Synchrotron radiation microtomography of Taylor bubbles in capillary two-phase flow," Exp. Fluids 55, 1 (2014).

${ }^{28}$ B. E. Ghidersa, M. Wörner, and D. G. Cacuci, "Exploring the flow of immiscible fluids in a square vertical mini-channel by direct numerical simulation," Chem. Eng. J. 101, 285 (2004).

${ }^{29}$ T. Taha and Z. F. Cui, "CFD modelling of slug flow inside square capillaries," Chem. Eng. Sci. 61, 665 (2006)

${ }^{30} \mathrm{M}$. Wörner, B. Ghidersa, and A. Onea, "A model for the residence time distribution of bubble-train flow in a square mini-channel based on direct numerical simulation results," Int. J. Heat Fluid Flow 28, 83 (2007).

${ }^{31}$ D. S. Liu and S. D. Wang, "Hydrodynamics of Taylor flow in noncircular capillaries," Chem. Eng. Process. 47, 2098 (2008)

${ }^{32}$ M. C. Öztaskin, M. Wörner, and H. S. Soyhan, "Numerical investigation of the stability of bubble train flow in a square minichannel," Phys. Fluids 21, 042108 (2009).

${ }^{33}$ A. Onea, M. Wörner, and D. G. Cacuci, "A qualitative computational study of mass transfer in upward bubble train flow through square and rectangular mini-channels," Chem. Eng. Sci. 64, 1416 (2009).

${ }^{34}$ S. Kececi, M. Wörner, A. Onea, and H. S. Soyhan, "Recirculation time and liquid slug mass transfer in co-current upward and downward Taylor flow," Catal. Today 147, S125 (2009).

${ }^{35}$ O. Keskin, M. Wörner, H. S. Soyhan, T. Bauer, O. Deutschmann, and R. Lange, "Viscous co-current downward Taylor flow in a square mini-channel," AIChE J. 56, 1693 (2010).

${ }^{36}$ M. Wörner, "Computational study on scaling of co-current downward Taylor flow in small square channels of three different sizes,” J. Chem. Eng. Jpn. 46, 335 (2013)

${ }^{37}$ H. Marschall, S. Boden, C. Lehrenfeld, C. J. D. Falconi, U. Hampel, A. Reusken, M. Wörner, and D. Bothe, "Validation of interface capturing and tracking techniques with different surface tension treatments against a Taylor bubble benchmark problem," Comput. Fluids 102, 336 (2014).

38 P. Angeli and A. Gavriilidis, "Hydrodynamics of Taylor flow in small channels: A review," Proc. Inst. Mech. Eng., Part C 222, 737 (2008).

${ }^{39}$ R. Gupta, D. F. Fletcher, and B. S. Haynes, "Taylor flow in microchannels: A review of experimental and computational work,” J. Comput. Multiphase Flows 2, 1 (2010).

${ }^{40}$ P. Sobieszuk, J. Aubin, and R. Pohorecki, "Hydrodynamics and mass transfer in gas-liquid flows in microreactors," Chem. Eng. Technol. 35, 1346 (2012).

${ }^{41}$ V. Talimi, Y. S. Muzychka, and S. Kocabiyik, "A review on numerical studies of slug flow hydrodynamics and heat transfer in microtubes and microchannels," Int. J. Multiphase Flow 39, 88 (2012).

${ }^{42}$ S. Aland, C. Lehrenfeld, H. Marschall, C. Meyer, and S. Weller, "Accuracy of two-phase flow simulations: The Taylor flow benchmark," PAMM 13, 595 (2013).

${ }^{43}$ S. Aland, S. Boden, A. Hahn, F. Klingbeil, M. Weismann, and S. Weller, "Quantitative comparison of Taylor flow simulations based on sharp-interface and diffuse-interface models," Int. J. Numer. Methods Fluids 73, 344 (2013).

${ }^{44}$ C. Meyer, M. Hoffmann, and M. Schlüter, "Micro-PIV analysis of gas-liquid Taylor flow in a vertical oriented square shaped fluidic channel," Int. J. Multiphase Flow 67, 140 (2014).

${ }^{45}$ S. P. Quan, "Co-current flow effects on a rising Taylor bubble," Int. J. Multiphase Flow 37, 888 (2011).

${ }^{46}$ M. Rieber, "Numerische modellierung der dynamik freier grenzflächen in zweiphasenströmungen," Fortschrittberichte VDI/7, VDI-Verlag, 2003.

${ }^{47}$ R. B. DeBar, "Fundamentals of the KRAKEN code. [Eulerian hydrodynamics code for compressible nonviscous flow of several fluids in two-dimensional (axially symmetric) region]," Technical Report UCID-17366 Lawrence Livermore Lab. CA, 1974.

${ }^{48}$ C. W. Hirt and B. D. Nichols, "Volume of fluid (VOF) method for the dynamics of free boundaries," J. Comput. Phys. 39, 201 (1981).

49 W. J. Rider and D. B. Kothe, "Reconstructing volume tracking," J. Comput. Phys. 141, 112 (1998).

${ }^{50}$ D. Bothe, M. Kröger, and H.-J. Warnecke, "A VOF-based conservative method for the simulation of reactive mass transfer from rising bubbles," Fluid Dyn. Mater. Process. 7, 303 (2011).

${ }^{51}$ D. Bothe and S. Fleckenstein, "A volume-of-fluid-based method for mass transfer processes at fluid particles," Chem. Eng. Sci. 101, 283 (2013).

${ }^{52}$ C. Gotaas, P. Havelka, H. A. Jakobsen, H. F. Svendsen, M. Hase, N. Roth, and B. Weigand, "Effect of viscosity on droplet-droplet collision outcome: Experimental study and numerical comparison," Phys. Fluids 19, 102106 (2007).

${ }^{53}$ C. Focke and D. Bothe, "Direct numerical simulation of binary off-center collisions of shear thinning droplets at high Weber numbers," Phys. Fluids 24, 073105 (2012). 
${ }^{54}$ C. Albert, H. Raach, and D. Bothe, "Influence of surface tension models on the hydrodynamics of wavy laminar falling films in volume of fluid-simulations," Int. J. Multiphase Flow 43, 66 (2012).

${ }^{55} \mathrm{~W}$. Sabisch, "Dreidimensionale numerische simulation der dynamik von aufsteigenden einzelblasen und blasenschwärmen mit einer volume-of-fluid-methode," Ph.D. thesis, University Karlsruhe, 2000.

${ }^{56} \mathrm{~S}$. Groß and A. Reusken, "Finite element discretization error analysis of a surface tension force in two-phase incompressible flows," SIAM J. Numer. Anal. 45, 1679 (2007).

${ }^{57}$ S. Groß, J. Peters, V. Reichelt, and A. Reusken, The DROPS package for numerical simulations of incompressible flows using parallel adaptive multigrid techniques, RWTH Aachen University, 2002, IGPM Report No. 211.

${ }^{58}$ E. Bertakis, S. Gross, J. Grande, O. Fortmeier, A. Reusken, and A. Pfennig, "Validated simulation of droplet sedimentation with finite-element and level-set methods," Chem. Eng. Sci. 65, 2037 (2010).

${ }^{59}$ M. Wörner, B. Ghidersa, and A. Shahab, Numerical Study of Bubble Train Flow in a Square Vertical Mini-Channel: Influence of the Length of the Flow Unit Cell (Proc. 5th Int. Conf. Multiphase Flow ICMF-2004, Yokohama, Japan, 2004).

${ }^{60}$ M. R. Ozgu, J. C. Chen, and A. H. Stenning, "Local liquid film thickness around Taylor bubbles," J. Heat Transfer 95, 425 (1973).

${ }^{61}$ L. W. Schwartz, H. M. Princen, and A. D. Kiss, "On the motion of bubbles in capillary tubes," J. Fluid Mech. 172, 259 (1986).

${ }^{62}$ E. Klaseboer, R. Gupta, and R. Manica, "An extended Bretherton model for long Taylor bubbles at moderate capillary numbers," Phys. Fluids 26, 032107 (2014).

${ }^{63}$ W. B. Kolb and R. L. Cerro, "Coating the inside of a capillary of square cross section," Chem. Eng. Sci. 46, 2181 (1991).

${ }^{64}$ A. L. Hazel and M. Heil, "The steady propagation of a semi-infinite bubble into a tube of elliptical or rectangular crosssection,” J. Fluid Mech. 470, 91 (2002).

${ }^{65}$ G. I. Taylor, "Deposition of a viscous fluid on the wall of a tube," J. Fluid Mech. 10, 161 (1961).

${ }^{66}$ B. G. Cox, "An experimental investigation of the streamlines in viscous fluid expelled from a tube," J. Fluid Mech. 20, 193 (1964).

${ }^{67}$ R. S. Abiev, "Bubbles velocity Taylor circulation rate and mass transfer model for slug flow in milli- and microchannels," Chem. Eng. J. 227, 66 (2013).

${ }^{68}$ B. K. H. Yen, A. Günther, M. A. Schmidt, K. F. Jensen, and M. G. Bawendi, "A microfabricated gas-liquid segmented flow reactor for high-temperature synthesis: The case of CdSe quantum dots," Angew. Chem., Int. Ed. 44, 5447 (2005).

${ }^{69}$ J. Hua, L. E. Erickson, T.-Y. Yiin, and L. A. Glasgow, "A review of the effects of shear and interfacial phenomena on cell viability," Crit. Rev. Biotechnol. 13, 305 (1993).

${ }^{70}$ M. Mercier, C. Fonade, and C. Lafforgue-Delorme, "How slug flow can enhance the ultrafiltration flux in mineral tubular membranes," J. Membr. Sci. 128, 103 (1997).

${ }^{71}$ N. Ratkovich, C. C. V. Chan, P. R. Berube, and I. Nopens, "Experimental study and CFD modelling of a two-phase slug flow for an airlift tubular membrane," Chem. Eng. Sci. 64, 3576 (2009).

72 T. Taha and Z. F. Cui, "CFD modelling of slug flow in vertical tubes," Chem. Eng. Sci. 61, 676 (2006).

${ }^{73}$ R. A. S. Brown and G. W. Govier, "Mechanics of large gas bubbles in tubes: II. Prediction of voidage in verticle gas-liquid flow," Can. J. Chem. Eng. 43, 224 (1965).

${ }^{74}$ R. S. Abiev, "Simulation of the slug flow of a gas-liquid system in capillaries," Theor. Found. Chem. Eng. 42, 105 (2008).

75 P. Aussillous and D. Quere, "Quick deposition of a fluid on the wall of a tube," Phys. Fluids 12, 2367 (2000). 